\title{
Shear sum rules at finite chemical potential
}

\author{
Justin R. David ${ }^{a}$, Sachin Jain ${ }^{b, c}$ and Somyadip Thakur ${ }^{a}$. \\ ${ }^{a}$ Centre for High Energy Physics, Indian Institute of Science, \\ C.V. Raman Avenue, Bangalore 560012, India. \\ justin, somyadip@cts.iisc.ernet.in \\ ${ }^{b}$ Tata Institute for Fundamental Research \\ Homi Bhabha Road, Mumbai 400005, India. \\ ${ }^{c}$ Institute of Physics, Sachivalaya Marg, \\ Bhubaneswar 751005, India. \\ sachin@theory.tifr.res.in
}

\begin{abstract}
We derive sum rules which constrain the spectral density corresponding to the retarded propagator of the $T_{x y}$ component of the stress tensor for three gravitational duals. The shear sum rule is obtained for the gravitational dual of the $\mathcal{N}=4$ Yang-Mills, theory of the M2-branes and M5-branes all at finite chemical potential. We show that at finite chemical potential there are additional terms in the sum rule which involve the chemical potential. These modifications are shown to be due to the presence of scalars in the operator product expansion of the stress tensor which have non-trivial vacuum expectation values at finite chemical potential.
\end{abstract}




\section{Contents}

1. Introduction 1

2. Sum rule generalities 3

3. The shear sum rule for uncharged D3-branes 5

3.1 Green's function from gravity 5

3.2 Green's function in the $\omega$-plane

3.3 The sum rule 17

3.4 Sum rule from OPE 17

4. The shear sum rule for the R-charged D3-brane 18

4.1 Green's function from gravity 19

4.2 Green's function in the $\omega$-plane 21

4.3 The sum rule 27

4.4 Sum rule from OPE 28

5. Shear sum rule for R-charged M2 and M5-branes 31

5.1 M2-branes 32

5.2 M5-branes 38

6. Conclusions 41

®. Fefferman-Graham coordinates 42

\section{Introduction}

Sum rules play an important role in constraining spectral densities of strongly coupled fluids. There are important sum rules which constrain spectral densities of stress tensor in QCD [1, 2, 3, 4, 5] and the Ferrell-Glover-Tinkham sum rule satisfied by current-current correlation function in the BCS superconductor [6, 7]. The gauge gravity duality provides a framework for evaluating the two point functions of various conserved currents of strongly coupled theories which admit a gravity dual. Thus one can use this framework to obtain sum rules. Obtaining sum rules within this frame work provides information of the analytic structure of the Green's functions in the strongly coupled limit. Romatschke and Son [8] derived two sum rules for the stress 
tensor two point function for strongly coupled $\mathcal{N}=4$ Yang-Mills theory. This was then generalized for Chamblin-Reall backgrounds which are dual to non-conformal theories in [9, 10]. The sum rule for the R-charge correlator in strongly coupled $\mathcal{N}=4$ Yang-Mills was obtained in [1].

As emphasized in [11], sum rules are the consequences of analyticity of the Green's function in the complex frequency plane. From the field theory point of view this results from the unitarity and causality of the field theory. Deriving sum rules from gravity provides insight into how the properties of unitarity and causality of the boundary field theory are encoded in the gravitational theory. In [1] the differential equations which determine the retarded Green's function of interest from gravity were studied. The properties of these differential equations were used to obtain proof and provide a unified framework to obtain various sum rules in gravity.

In this paper we obtain the sum rules from gravity for the spectral density corresponding to the retarded propagator of the $T_{x y}$ component of the stress tensor. This is done for the case of $\mathcal{N}=4$ Yang-Mills, the M2-brane and the M5-brane theory in the presence of chemical potential. One of our motivations to examine these situations is to determine how the sum rules are modified when the dual theory cannot be truncated to pure gravity. The presence of chemical potentials in these systems results in additional scalars in the gravitational theory. We will now briefly state the result for the $\mathcal{N}=4$ Yang-Mills case. The shear sum rule for $\mathcal{N}=4$ Yang-Mills derived in [8] in the absence of chemical potential is given by

$$
\frac{2}{5} \epsilon=\frac{1}{\pi} \int_{-\infty}^{\infty} \frac{d \omega}{\omega}\left(\rho(\omega)-\rho_{T=0}(\omega)\right),
$$

where

$$
\rho=\operatorname{Im} G_{R}(\omega)
$$

and $G_{R}$ is the retarded propagator of the $T_{x y}$ component of the stress tensor. $\rho_{T=0}(\omega)$ is the spectral density at zero temperature and $\epsilon$ is the finite temperature energy density. In this paper we examine this sum rule in the presence of chemical potential. We find that the sum rule is modified to

$$
\frac{2}{5} \epsilon-\frac{N^{2} \pi^{2} T_{0}^{4}}{120}\left\{\left(k_{1}-k_{2}\right)^{2}+\left(k_{1}-k_{3}\right)^{2}+\left(k_{2}-k_{3}\right)^{2}\right\}=\frac{1}{\pi} \int_{-\infty}^{\infty} \frac{d \omega}{\omega}\left(\rho(\omega)-\rho_{T=0}(\omega)\right) .
$$

Where $k_{i}^{\prime}$ s are functions of the three chemical potentials in $\mathcal{N}=4$ Yang-Mills given in (4.6). The relation between $T_{0}$ and the temperature of the Yang-Mills is given by (4.6). Note that for the situation when all the charges or chemical potentials are equal then the correction vanishes. For this case the the gravity background has no additional scalars present. We then show that these additional terms in the sum rule are due to the fact that, the operator product expansion of two stress tensor involves operators of dimension 4 in addition to the stress tensor. We show that 
expectation values of these operators precisely have the same dependence in terms of the chemical potential to account for the additional terms.

For the M2-brane theory, the shear sum rule at finite chemical potential is given by

$$
\begin{aligned}
& \frac{3}{8} \epsilon+\frac{\sqrt{2} \pi^{2} N^{3 / 2} T_{0}^{3}}{216}\left(k_{1}+k_{2}-k_{3}-k_{4}\right)\left(k_{1}-k_{2}+k_{3}-k_{4}\right)\left(k_{1}-k_{2}-k_{3}+k_{4}\right) \\
& =\frac{1}{\pi} \int_{-\infty}^{\infty} \frac{d \omega}{\omega}\left(\tilde{\rho}(\omega)-\rho_{T=0}(\omega)\right) .
\end{aligned}
$$

Note that for the case of the M2-brane theory, there are 4-chemical potentials which are related to the $k_{i}$ 's by (5.9) and $T_{0}$ is related to the temperature by (5.8). $\tilde{\rho}$ is the imaginary part of the retarded Green's function shifted by a term which is linear in frequency defined in (5.24). The term proportional to the energy density arises from the expectation value of the stress tensor in the OPE, while the term proportional to the charge density arises from expectation value of scalars. Finally for the M5-brane theory, the shear sum rule at chemical potential remains unchanged in the presence of chemical potential. It is given by

$$
\frac{3}{7} \epsilon=\frac{1}{\pi} \int_{-\infty}^{\infty} \frac{d \omega}{\omega}\left(\rho(\omega)-\rho_{T=0}(\omega)\right) .
$$

In this case we show the scalars which are turned on due to the chemical potential do not have the appropriate conformal dimensions to occur in the OPE of the stress tensor and modify the sum rule.

The organization of the paper is as follows. In the next section we discuss the general considerations which go into deriving sum rules in quantum field theories. In section 3. we review the proof of the sum rule given in [11] for the case of $\mathcal{N}=4$ Yang-Mills in the absence of chemical potentials. Here we develop a method to obtain the behaviour of the retarded Green's function at large frequencies using the

Fefferman-Graham coordinates. This method also allows us to extract the LHS of the sum rule easily. In section 4 . we derive the sum rule for D3-branes at finite chemical potential and explain the occurrence of additional terms in the sum rule (1.3) due to the presence of expectation values of scalars. In section 5. we derive the sum rule for the M2 and M5-brane theories. Section 6. contains our conclusions. Appendix A contains the details of the Fefferman-Graham coordinates which are used to derive the sum rule.

\section{Sum rule generalities}

Consider the retarded Greens function corresponding to an operator $\mathcal{O}$ of a quantum field theory in 4 dimensions given by

$$
\tilde{G}_{R}(t, x) \equiv i \theta(t)\langle[\mathcal{O}(t, x), \mathcal{O}(0,0)]\rangle
$$


we then take its Fourier transform given by

$$
G_{R}(\omega, k)=\int d^{4} x e^{i(\omega t-i k x)} \tilde{G}_{R}(t, x) .
$$

The spectral density corresponding to this retarded correlator is defined as

$$
\rho(\omega, k)=\frac{1}{2 i}\left\{G_{R}(\omega, k)-G_{R}(\omega, k)^{*}\right\} .
$$

It is easy to see that from (2.1) and from (2.2) we see that for bosonic Hermitian operators we get

$$
G_{R}(\omega, k)^{*}=G_{R}(-\omega,-k) .
$$

In this paper we will restrict out attention to the case $k=0$ and we define $G_{R}(\omega)=$ $G_{R}(\omega, 0)$. From the reality property (2.4), we see that in the Taylor series expansion of $G_{R}(\omega)$ even powers of $\omega$ have real coefficients and odd powers of $\omega$ have purely imaginary coefficients. Sum rules for the spectral density are obtained by assuming certain analytic properties of the Green's function in the complex $\omega$-plane.

The properties are the following

1. $G_{R}(\omega)$ is holomorphic in the upper half plane, including the real axis.

2. $\lim _{|\omega| \rightarrow \infty} G_{R}(\omega)=0$ if $\operatorname{Im} \omega \geq 0$.

These properties will be referred to as property 1 and property 2 in the rest of the paper. From the field theory point of view causality ensures property 1 is satisfied. Using these properties, we now indicate the arguments which go into deriving the sum rule. By the first property and by Cauchy's theorem we have

$$
\begin{aligned}
G_{R}(\omega+i \epsilon) & =\frac{1}{2 \pi i} \oint_{C} \frac{G_{R}(z) d z}{z-\omega-i \epsilon} \\
0 & =\frac{1}{2 \pi i} \oint_{C} \frac{G_{R}(z) d z}{z-\omega+i \epsilon} .
\end{aligned}
$$

for $\omega, \epsilon \in R$ and $\epsilon>0$. The contour $C$ is chosen such that it runs from $-r$ to $r$ for some large $r$ and then along the semi-circle in the upper half plane and back to $-r$ with $r>0$. Because of the second property the integrals along the contour in (2.5) reduce to

$$
\begin{aligned}
G_{R}(\omega+i \epsilon) & =\frac{1}{2 \pi i} \lim _{r \rightarrow \infty} \int_{-r}^{r} \frac{G_{R}(z) d z}{z-\omega-i \epsilon} \\
0 & =\frac{1}{2 \pi i} \lim _{r \rightarrow \infty} \int_{-r}^{r} \frac{G_{R}(z) d z}{z-\omega+i \epsilon} .
\end{aligned}
$$

Then taking the difference of the two equations in (2.6) we obtain

$$
G_{R}(\omega)=\lim _{\epsilon \rightarrow 0^{+}} \int_{-\infty}^{\infty} \frac{d z}{\pi} \frac{\rho(z)}{z-\omega-i \epsilon},
$$


where we have used the definition of the spectral density. Note that the integral runs over the real line. The usual sum rule is obtained by evaluating the left hand side of (2.7) at $\omega=0$. Thus we have

$$
G_{R}(0)=\lim _{\epsilon \rightarrow 0^{+}} \int_{-\infty}^{\infty} \frac{d \omega}{\pi} \frac{\rho(\omega)}{\omega-i \epsilon}
$$

In many of the situations the Green's functions may not satisfy property 2 , that is $\lim _{|\omega| \rightarrow \infty} G_{R}(\omega)=0$ if $\operatorname{Im} \omega \geq 0$. In such situations we subtract divergences and obtain a regulated Green's function so that we can still ensure that property 2 will be satisfied. The two point function of the stress tensor which we will study in this paper falls into this case.

\section{The shear sum rule for uncharged D3-branes}

In this section we review the derivation of the shear sum rule from gravity for $\mathcal{N}=$ 4 Yang-Mills at zero chemical potential. This will help us set up notations and conventions. To obtain the retarded Green's function of the $T_{x y}$ component of the stress tensor one needs to solve the equation of the minimally coupled scalar field in the background of the D3-branes [12, 13]. Examining this differential equation it is possible to argue that property 1 is satisfied. To ensure property 2, we will

develop a method to obtain the behaviour of the Green's function in gravity at large frequencies. This will enable us to regulate the Green's function and ensure property 2 , which will lead us to the derivation of the sum rule.

\subsection{Green's function from gravity}

As we have mentioned earlier the retarded Green's function of interest in this paper is given by

$$
G_{R}(t, \vec{x})=i \theta(t)\left\langle\left[T_{x y}(t, \vec{x}), T_{x y}(0,0)\right]\right\rangle,
$$

where $T_{x y}$ is the $x y$ component of the stress tensor. The dual geometry corresponding to $\mathcal{N}=4$ Yang-Mills at finite temperature is the non-extremal D3-brane background. The metric of this background is given by

$$
\begin{aligned}
d s^{2} & =\frac{r^{2}}{L^{2}}\left(-f d t^{2}+d x^{2}+d y^{2}+d z^{2}\right)+\frac{L^{2}}{r^{2} f} d r^{2} \\
f & =1-\frac{r_{+}^{4}}{r^{4}}
\end{aligned}
$$

The above metric is a solution of the action

$$
S=\frac{N^{2}}{8 \pi^{2} L^{3}} \int d^{5} x \sqrt{g}\left(R+\frac{12}{L^{2}}\right) .
$$


It is useful to recall the thermodynamic variables of this system. The temperature, energy density, pressure and entropy density of this system are given by

$$
\begin{gathered}
T=\frac{r_{+}}{\pi L^{2}}, \quad \epsilon=\frac{3 \pi^{2} N^{2} T^{4}}{8}, \\
P=\frac{\pi^{2} N^{2} T^{4}}{8}, \quad s=\frac{\pi^{2} N^{2} T^{3}}{2} .
\end{gathered}
$$

To evaluate the Green's function given in (3.1) from the gravity dual we first consider the metric fluctuation which is dual to the stress tensor $T_{x y}$. This is given by

$$
\delta g_{x y}=\phi(r) e^{-i \omega t+i k z} \frac{r^{2}}{L^{2}}
$$

This perturbation obeys the equation of motion of a minimally coupled massless scalar in the background (3.2). The $k=0$ mode satisfies the following equation

$$
\partial_{r}^{2} \phi+\left(\frac{F^{\prime}}{F}+\frac{3}{r}\right) \partial_{r} \phi+\frac{\omega^{2}}{F^{2}} \phi=0,
$$

where

$$
F=\frac{r^{2}}{L^{2}} f
$$

The procedure to obtain the Green's function [14] is to first impose in going boundary conditions at the horizon $r_{+}$and obtain $\phi$ at the boundary $r \rightarrow \infty$. Once this is done, the Green's function at temperature $T$ is given by

$$
\begin{aligned}
G_{R}(\omega, T) & =\hat{G}_{R}(\omega, T)+G_{\text {contact }}(T)+G_{\text {counter }}(\omega, T), \\
\hat{G}_{R}(\omega) & =-\frac{N^{2}}{8 \pi^{2} L^{6}} \lim _{r \rightarrow \infty} \frac{F r^{3} \phi^{\prime}}{\phi}
\end{aligned}
$$

where $G_{\text {contact }}(T)$ is the contribution from the contact terms obtained from the on shell effective action. The on shell effective action is given by [11].

$$
S_{\mathrm{os}}=\left.P \int d^{4} x\left(1-\frac{1}{2} \phi^{2}\right)\right|_{r \rightarrow \infty},
$$

where $P$ is the pressure of the solution. Evaluating $G_{\text {contact }}$ we obtain

$$
G_{\text {contact }}=-P \text {. }
$$

$G_{\text {counter }}(\omega, T)$ is the contribution from the counter terms required to cancel the $r^{2}$ and $\log (r)$ divergences in $G_{R}(\omega)$. The details of these terms are given in [11], but as we will see later, we will not need the detailed structure of these terms. An important point to note is that the on-shell action is independent of frequency which ensures that the contact term $G_{\text {contact }}$ is also independent of frequency. 
Thus the important properties of the Green's function is essentially contained in the function

$$
g_{R}(\omega)=\lim _{r \rightarrow \infty} \frac{F r^{3} \phi^{\prime}}{\phi} .
$$

Therefore to study the behaviour of the retarded Green's function, it is sufficient to study the function $g_{R}(\omega)$ whose behaviour can be understood by examining the equation (3.6).

Before we go ahead, we first discuss the asymptotic properties of the solution of the differential equation (3.6). Near the horizon the solutions are wave like and is given by

$$
\phi(r) \sim\left(r-r_{+}\right)^{ \pm \frac{i \omega}{F_{h}}}, \quad r \rightarrow r_{+},
$$

where $F_{h}$ is given by the expansion

$$
F=\left(r-r_{+}\right) F_{h}+\cdots, \quad F_{h}=4 \frac{r_{+}}{L^{2}} .
$$

Now at the boundary the two independent solutions are given by

$$
\begin{aligned}
& \phi(r) \rightarrow \frac{L^{4} \omega^{2}}{r^{2}} J_{2}\left(\frac{L^{2} \omega}{r}\right) \sim r^{-4}, \quad r \rightarrow \infty, \\
& \phi(r) \rightarrow \frac{L^{4} \omega^{2}}{r^{2}} K_{2}\left(i \frac{L^{2} \omega}{r}\right) \sim \text { constant }, \quad r \rightarrow \infty .
\end{aligned}
$$

We will also need the fact that the differential equation given in (3.6) can be obtained as the equations of motion of the following action

$$
S_{\phi}=\int_{r_{h}}^{\infty} d r F r^{3}\left(\left|\phi^{\prime}(r)\right|^{2}-\frac{\omega^{2}}{F^{2}}|\phi(r)|^{2}\right) .
$$

\subsection{Green's function in the $\omega$-plane}

In this subsection we will discuss the analytic properties of the function $g_{R}(\omega)$ defined in (3.11) in the complex $\omega$-plane.

No poles for $\operatorname{Im} \omega>0$.

Poles or divergences $g_{R}(\omega)$ correspond to quasi-normal modes of the equation (3.6) [13]. Quasi-normal modes are solutions to the differential equation (3.6) with the following boundary conditions.

$$
\begin{aligned}
& \phi(r) \sim\left(r-r_{+}\right)^{-i \frac{\omega}{F_{h}}}, \quad r \rightarrow r_{+}, \\
& \phi(r) \sim r^{-4}, \quad r \rightarrow \infty .
\end{aligned}
$$

We will now prove that such quasi-normal modes do not exist. A simple intuitive reason that prohibits such quasi-normal modes is that, if quasi-normal modes with 
$\operatorname{Im} \omega>0$ exists, they will correspond to instabilities due to the time dependence $\exp (-i \omega t)$ in the mode given in (3.5). We will now demonstrate such quasi-normal modes do not exist directly from the equation (3.6). All the coefficients in the equation (3.6) are real. Therefore if $\phi(r)$ is a quasi-normal mode with say complex frequency $\omega$, then $\phi(r)^{*}$ the complex conjugate is also a quasi-normal mode with frequency $\omega^{*}$. This is seen by just taking the complex conjugate of the equation (3.6). We can now consider $S_{\phi}-S_{\phi}=0$ which by integration by parts and using equations of motion we obtain

$$
0=\left.F r^{3}\left(\phi^{* \prime} \phi-\phi^{*} \phi^{\prime}\right)\right|_{r_{h}} ^{\infty}+\left(\omega^{* 2}-\omega^{2}\right) \int_{r_{h}}^{\infty} d r \frac{r^{3}}{F}|\phi|^{2} .
$$

Here we have used the equations of motion of $\phi^{*}$ in the first $S_{\phi}$ and the equation of motion of $\phi$ in the second $S_{\phi}$. Now from the conditions for the quasi-normal modes given in (3.16) and the fact that $F \sim r^{2}$ as $r \rightarrow \infty$ and $F \sim\left(r-r_{h}\right) F_{h}$ as $r \rightarrow r_{h}$ we see that the first term in the above equation vanishes for $\operatorname{Im}(\omega)>0$. Then we have the case that

$$
\omega^{2}=\omega^{* 2},
$$

since the second term is positive definite. Thus we see that $\omega$ has to be either purely real or purely imaginary, now with the condition $\operatorname{Im}(\omega)>0$ we find that $\omega$ is restricted to be on the upper imaginary axis.

Let us now assume that there exists a quasi normal modes on the upper imaginary axis with $\omega^{2}<0$. Then $S_{\phi}$ is positive definite. Substituting this solution in $S_{\phi}$ and integrating by parts we obtain

$$
S_{\phi}=\left.F r^{3} \phi^{*}(r) \phi^{\prime}(r)\right|_{r_{h}} ^{\infty} .
$$

This vanishes from the behaviour in (3.16) and the fact that $\operatorname{Im} \omega>0$. But since $S_{\phi}$ is positive definite we must have $\phi=0$. Thus no quasi-normal modes exist in the upper half plane which implies no poles or divergences exist in the Green's function in this domain.

No poles for $\omega$ real and $\omega \neq 0$

For real $\omega$, the following quantity

$$
W=\phi^{\prime} \phi^{*}-\phi^{\prime *} \phi
$$

is the Wronskian. From the differential equation in (3.6) we see that the Wronskian satisfies the equation

$$
W^{\prime}+\left(\frac{F^{\prime}}{F}+\frac{3}{r}\right) W=0 .
$$


The solution of this equation is given by

$$
W=\frac{C}{r^{3} F} .
$$

We can determine the constant $C$ since we know that if there exists a quasi-normal mode, the behaviour of the mode near the horizon is given by the ingoing boundary conditions given in (3.16). This fixes the $C$ which determines the Wronskian to be given by

$$
W=-i \frac{2 \omega r_{+}^{3}}{r^{3} F} .
$$

From here we see that the quantity $r^{3} F W$ does not vanish any where. We can now examine its behaviour as $r \rightarrow \infty$ for the quasi-normal mode. The boundary conditions for the quasi-normal mode given in (3.16) implies that $\phi \rightarrow r^{-4}$ as $r \rightarrow \infty$. Using this fact in the definition given in (3.20) we conclude the quantity $r^{3} F W$ must vanish as $r \rightarrow \infty$ which contradicts our previous conclusion. Thus quasi-normal modes and hence poles or divergences of the Greens's function do not exist on the real line for $\omega \neq 0$.

\section{No poles for $\omega=0$}

We can determine the behaviour of the function $g_{R}$ as $\omega \rightarrow 0$ explicitly from the differential equation (3.6) as follows. Let us define

$$
\tilde{g}(r)=\frac{\phi^{\prime}(r)}{\omega \phi(r)} .
$$

We can use the differential equation (3.6) to obtain the equation satisfied by $\tilde{g}$. This is given by

$$
\tilde{g}^{\prime}(r)+\omega \tilde{g}^{2}(r)+\left(\frac{F^{\prime}}{F}+\frac{3}{r}\right) \tilde{g}(r)+\frac{\omega}{F^{2}}=0 .
$$

The ingoing boundary conditions at the horizon given in (3.16) determines the boundary condition for $\tilde{g}$, this is given by

$$
\tilde{g} \sim \frac{-i}{F_{h}\left(r-r_{+}\right)}, \quad r \rightarrow r_{+} .
$$

The equation (3.25) can be easily solved in the $\omega \rightarrow 0$ limit. The solution which agrees with the boundary condition in (3.26) is given by

$$
\tilde{g}=-i \frac{r_{+}^{3}}{r^{3} F}, \quad \omega \rightarrow 0 .
$$

This implies

$$
g_{R}=\omega \lim _{r \rightarrow \infty} r^{3} F \tilde{g}=-i \omega r_{+}^{3}, \quad \omega \rightarrow 0 .
$$


Thus we conclude that the Green's function has no poles as $\omega \rightarrow 0$. In fact as can be seen from the equation (3.25), it admits an analytic power series expansion in $\omega$ at the origin. The leading behaviour is given above in (3.28). Using (3.25) one can easily set up a perturbative expansion in $\omega$ and solve for $g_{R}(\omega)$ order by order in $\omega$ which gives rise to the power series expansion around the origin.

No zero's for $\operatorname{Im} \omega \geq 0, \omega \neq 0$

Just as quasi-normal modes correspond to Dirichlet boundary conditions for the field $\phi$ at the boundary which leads to the boundary conditions given in (3.16) zeros of the Green's function correspond to Neumann boundary conditions for the field $\phi$. This can be seen from the definition (3.11) which implies that zeros of the Green's function correspond to the condition

$$
\begin{aligned}
\phi(r) & \sim\left(r-r_{+}\right)^{-i \frac{\omega}{F_{h}}}, \quad r \rightarrow r_{+}, \\
r^{3} F \phi^{\prime}(r) & \rightarrow 0, \quad r \rightarrow \infty .
\end{aligned}
$$

One might suspect that there might be singularities in $\phi$ which also gives zeros of the Green's function. However as discussed in [13], a theorem of [15] guarantees that the solution is smooth with respect to a parameter $\omega$ if the differential equation and the boundary conditions are smooth with respect to it. This ensures that there cannot be singularities in $\phi$ in the $\omega$ plane. Now with the Neumann boundary conditions given in (3.29) we can repeat the steps of the discussion for the absence of poles or singularities in the Green's function. All the steps goes through since, here again the boundary conditions in (3.29) ensures that

$$
F r^{3}\left(\phi^{* \prime} \phi-\phi^{*} \phi^{\prime}\right)=0, \quad r \rightarrow r_{+}, r \rightarrow \infty .
$$

Similarly for $\operatorname{Im} \omega=0, \omega \neq 0$ we have the limit

$$
W F r^{3} \rightarrow 0, \quad r \rightarrow \infty,
$$

where $W$ is the Wronskian. These conditions allows us to conclude that the Green's function does not vanish in the upper half $\omega$ plane including the real axis, with the exception of $\omega=0$ where we have shown that it vanishes and it admits an analytic power series expansion.

\section{Absence of branch cuts for $\operatorname{Im} \omega \geq 0^{1}$}

Recall that the important properties of the Green's function is essentially contained in the function $g_{R}(\omega)$ as defined in (3.11). In this equation $\phi(r)$ is the solution

\footnotetext{
${ }^{1}$ We would like to thank Ashoke Sen and the anonymous referee of this paper for useful comments on this section.
} 
for the minimally coupled scalar equation in the background of (3.2) with ingoing boundary (4.14) at the horizon. From (3.11) we see that that we need to examine the behaviour of solution $\phi(r)$ and its derivative at the boundary. Note that the differential equation for the minimally coupled scalar and the boundary condition depends on the parameter $\omega$ and both these dependences are smooth with respect to this parameter $\omega$. Now the general theorem in [15] ${ }^{2}$ ensures that the local Forbenius expansion of the solution is smooth with respect to the parameters of the differential equation if both the equation and the boundary condition are smooth with respect to the parameter. Hence the Forbenius expansion of $\phi$ and its radial derivative at the boundary are smooth with respect to the parameter $\omega$. Now using this theorem it is easy to see that the $n^{\text {th }}$ order derivative of the $g_{R}(\omega)$ with respect to $\omega$ is smooth in the upper half $\omega$ plane. Since $\phi$ and its derivative are smooth with respect to $\omega$ we see from (3.11) the only possible locations of singularities of the $n^{\text {th }}$ order derivative of the $g_{R}(\omega)$ are the zeros of $\phi$, that is where it satisfies the quasi-normal mode boundary conditions given in (3.16). However we have shown earlier that there are no-quasi-normal modes in the upper half-plane, this assures that arbitrary derivatives of $g_{R}(\omega)$ exists in the upper half plane. This implies that the Green's function does not have any branch cuts in the upper half-plane.

Thus we have concluded that the Green's function satisfies property 1 for the derivation of the sum rule. We now need to ensure that property 2 is satisfied.

\section{Behaviour as $\omega \rightarrow \infty$}

To obtain the behaviour of the Green's function at large $\omega$ we first rewrite the differential equation given in (3.6) by defining a dimensionless variables

$$
z=\frac{r_{+}}{r}, \quad i \lambda=\frac{L^{2}}{r_{+}} \omega .
$$

The equation reduces to

$$
\phi^{\prime \prime}(z)-\frac{1}{z f(z)}\left(3+z^{4}\right) \phi^{\prime}(z)-\frac{\lambda^{2}}{f(z)^{2}} \phi(z)=0,
$$

where $f(z)=1-z^{4}$. Note that for convenience we have gone over to the Euclidean frequency labelled by $\lambda$. Let us re-state the boundary conditions in terms of these coordinates. The ingoing boundary condition reduces to

$$
\phi(z) \sim(1-z)^{\frac{\lambda}{4}}, \quad z \rightarrow 1
$$

\footnotetext{
${ }^{2}$ In fact this theorem has been used in the identification of the location of the poles of the Green's function with the quasi-normal modes of $\phi$ in 13 . Since in general the poles of the Green's function can also arise from the singularities of $\phi^{\prime}$. However this theorem guarantees that $\phi^{\prime}$ is smooth with respect to $\omega$ hence poles can arise only from the zeros of $\phi$ which correspond to its quasi-normal modes.
} 
and the Green's function is given by

$$
g_{R}(\lambda)=-\frac{r_{+}^{4}}{L^{2}} \lim _{z \rightarrow 0} \frac{\phi^{\prime}}{z^{3} \phi}
$$

Our aim now is to find the behaviour of this function as $\lambda \rightarrow \infty$. For this purpose we first rescale the co-ordinates as

$$
y=\lambda z
$$

The differential equation given in (3.33) can be written as

$$
\phi^{\prime \prime}(y)-\frac{1}{y f(y)}\left(3+\frac{y^{4}}{\lambda^{4}}\right) \phi^{\prime}(y)-\frac{1}{f(y)^{2}} \phi(y)=0
$$

where $f=1-\frac{y^{4}}{\lambda^{4}}$ and derivatives are with respect to $y$. We can solve the equation using the Forbenius expansion at around $y=\lambda$. This results in following series

$$
\phi=\left(1-\frac{y}{\lambda}\right)^{\frac{\lambda}{4}}\left(\sum_{j=0}^{\infty} a_{j}\left(1-\frac{y}{\lambda}\right)^{j}\right)
$$

The coefficients $a_{j}$ can be determined by recursion relations on substituting the Forbenius expansion in the differential equation (3.37). Now the differential equation in (3.37) also admits an expansion at around $y \rightarrow 0$. This expansion can be organized as a systematic expansion in powers of $\frac{1}{\lambda}$ as follows: We first expand (3.37) in powers of $1 / \lambda$, this results in

$$
\phi^{\prime \prime}(y)-\frac{1}{y}\left(3+4 \frac{y^{4}}{\lambda^{4}}+4 \frac{y^{8}}{\lambda^{8}}+\cdots\right) \phi^{\prime}(y)-\left(1+2 \frac{y^{4}}{\lambda^{4}}+3 \frac{y^{8}}{\lambda^{8}}+\cdots\right) \phi(y)=0 .
$$

One important fact to point out in this expansion is that at $\lambda$ strictly infinity, the equation reduces to that of the minimally coupled equation in the background of pure $A d S_{5}$, that is the background corresponding to zero temperature. To solve this equation perturbatively in $\frac{1}{\lambda}$ we define the quantity

$$
g(y)=\frac{\phi^{\prime}(y)}{\phi(y)}
$$

From the expansion of the equation in (3.39), we see that the we can expand $g$ as

$$
\begin{aligned}
g & =g_{0}(y)+\frac{1}{\lambda^{4}} g_{1}(y)+\frac{1}{\lambda^{8}} g_{2}(y)+\cdots \\
& =\sum_{n=0}^{\infty} \frac{1}{\lambda^{4 n}} g_{n} .
\end{aligned}
$$


We can obtain the equations satisfied by each $g_{n}$ by substituting the expansion above and matching orders in $\frac{1}{\lambda^{4}}$. The first few equations are given by

$$
\begin{aligned}
& g_{0}^{\prime}(y)+g_{0}^{2}(y)-\frac{3}{y} g_{0}(y)-1=0, \\
& g_{1}^{\prime}(y)+\left(2 g_{0}(y)-\frac{3}{y}\right) g_{1}(y)-4 y^{3} g_{0}(y)-2 y^{4}=0, \\
& g_{2}^{\prime}(y)+\left(2 g_{0}(y)-\frac{3}{y}\right) g_{2}(y)+g_{1}^{2}(y)-4 y^{3} g_{1}(y)-4 y^{7} g_{0}(y)-3 y^{8}=0 .
\end{aligned}
$$

The advantage of casting the equations in this form is that the apart form the first equation, the subsequent equations are first order equations. We will now illustrate how to obtain the two independent solutions. There are two solutions to the first equation, these are given by

$$
g_{0}^{(1)}=-\frac{K_{1}(y)}{K_{2}(y)}=\frac{d}{d y}\left(\log \left(y^{2} K_{2}(y)\right)\right), \quad g_{0}^{(2)}=\frac{I_{1}(y)}{I_{2}(y)}=\frac{d}{d y}\left(\log \left(y^{2} I_{2}(y)\right)\right) \text {. }
$$

Let us first find the $\frac{1}{\lambda}$ expansion around the first solution, for that we have to solve for $g_{1}$, this is given by

$$
\begin{aligned}
g_{1}^{(1)}(y) & =\frac{1}{y K_{2}^{2}(y)} \int_{0}^{y} d y\left[4 y^{3} g_{0}^{(1)}(y)+2 y^{4}\right]\left(y K_{2}^{2}(y)\right)+\frac{c_{1}}{y K_{2}^{2}(y)} \\
& =2 y^{3}+\frac{y^{5}}{5}\left(1-\frac{K_{3}^{2}}{K_{2}^{2}}\right)+\frac{c_{1}}{y K_{2}^{2}(y)}
\end{aligned}
$$

The term proportional to $c_{1}$ is the homogeneous solution. The constant $c_{1}$ is set to zero by requiring that the asymptotics of the solution set by $g_{0}$ be unchanged in the presence of $g_{1}$. Note that if $c_{1} \neq 0, g_{1}$ grows exponentially when $y \rightarrow \infty$. This condition is imposed on all equations. Thus we have

$$
\begin{aligned}
g_{1}^{(1)}(y) & =2 y^{3}+\frac{y^{5}}{5}\left(1-\frac{K_{3}^{2}}{K_{2}^{2}}\right), \\
& =-\frac{6}{5} y^{3}-\frac{3}{5} y^{5}+O\left(y^{7}, y^{7} \log (y)\right) .
\end{aligned}
$$

Similarly the solution of $g_{2}$ is given by

$$
g_{2}(y)=\frac{1}{y K_{2}^{2}(y)} \int_{0}^{y} d y\left[-\left(g_{1}^{(1)}\right)^{2}(y)+4 y^{3} g_{1}^{(1)}(y)+4 y^{7} g_{0}^{(1)}(y)+3 y^{8}\right]\left(y K_{2}^{2}(y)\right)
$$

From the expansion of the functions involved, it can be shown that $g_{2}^{(1)}$ admits the following expansion near the origin

$$
g_{2}^{(1)}(y) \sim-\frac{156}{25} y^{7}+y^{9}+(\log (y)+\log (2)+\gamma) y^{11}+\cdots
$$


An simple examination of the equation for $g_{n}$ shows that the leading term in the expansion of $g_{n}^{(1)}$ around the origin is given by

$$
g_{n}^{(1)}(y) \sim y^{m}, \quad m \geq 7, \quad \text { for } n \geq 2 .
$$

Now that we have $g_{n}$, the first solution $\left.\phi^{(1)}(y)\right)$ is given by

$$
\begin{aligned}
\phi^{(1)}(y)= & \exp \left(\int_{0}^{y} d y \sum_{n=0}^{\infty} \frac{g_{n}}{\lambda^{4 n}}\right), \\
= & y^{2} K_{2}(y)\left(1+\frac{1}{\lambda^{4}} \int_{0}^{y} d y g_{1}^{(1)}(y)\right. \\
& \left.\left.+\frac{1}{\lambda^{8}} \frac{1}{2}\left[\int_{0}^{y} d y g_{1}^{(1)}(y)\right)^{2}+\int_{0}^{y} g_{2}^{(1)}(y)\right]+\cdots\right) .
\end{aligned}
$$

Note that $\phi^{(1)}$ has the following behaviour as $y \rightarrow 0$

$$
\phi^{(1)} \sim \text { constant }, \quad y \rightarrow 0 .
$$

From (3.45) we see that the $\frac{1}{\lambda^{4}}$ coefficient in the expansion given in (3.49) behaves as $y^{4}$ near the origin. The equation (3.48) shows that the remaining terms are further suppressed as $y \rightarrow 0$.

A similar construction can be done starting with the seed $g_{0}^{(2)}$. The first $\frac{1}{\lambda^{4}}$ expansion around the second solution is given by

$$
\begin{aligned}
& g_{1}^{(2)}(y)=\frac{1}{y I_{2}^{2}(y)} \int_{0}^{y} d y\left[4 y^{3} g_{0}^{(2)}(y)+2 y^{4}\right]\left(y I_{2}^{2}(y)\right), \\
& g_{1}^{(2)}(y)=2 y^{3}+\frac{y^{5}}{5}\left(1-\frac{I_{3}^{2}}{I_{2}^{2}}\right) .
\end{aligned}
$$

Note that we have set the constant corresponding to the homogeneous solution to zero. Similarly the solution of $g_{2}^{(2)}$ is given by

$$
g_{2}^{(2)}(y)=\frac{1}{y I_{2}^{2}(y)} \int_{0}^{y} d y\left[-\left(g_{1}^{(2)}\right)^{2}(y)+4 y^{3} g_{1}^{(2)}(y)+4 y^{7} g_{0}^{(2)}(y)+3 y^{8}\right]\left(y I_{2}^{2}(y)\right) .
$$

From the expansions of the functions involved it can be shown that

$$
g_{2}^{(2)}(y) \sim y^{7}, \quad y \rightarrow 0
$$

Similarly in general it can be shown that

$$
g_{n}^{(2)}(y) \sim y^{m}, \quad m \geq 7, \quad \text { for } n \geq 2, \quad y \rightarrow 0 .
$$

Thus we obtain the second solution

$$
\begin{aligned}
\phi^{(2)}(y)= & y^{2} I_{2}(y)\left(1+\frac{1}{\lambda^{4}} \int_{0}^{y} d y g_{1}^{(2)}(y)\right. \\
& \left.\left.+\frac{1}{\lambda^{8}} \frac{1}{2}\left[\int_{0}^{y} d y g_{1}^{(2)}(y)\right)^{2}+\int_{0}^{y} g_{2}^{(2)}(y)\right]+\cdots\right) .
\end{aligned}
$$


It will be useful to note that $\phi^{(2)}$ has the following behaviour as $y \rightarrow 0$.

$$
\phi^{(2)} \sim y^{4}, \quad y \rightarrow 0 .
$$

Note that from (3.51) and (3.54) we see that the coefficients of the $\frac{1}{\lambda}$ expansion are further suppressed as $y \rightarrow 0$.

Once the solutions $\phi^{(1)}, \phi^{(2)}$ have been constructed, then the solution which matches the ingoing boundary condition at the horizon is obtained as follows: Considering the linear combination

$$
\phi(y)=A(\lambda) \phi^{(1)}(y)+B(\lambda) \phi^{(2)}(y) .
$$

The coefficients $A, B$ which depend on $\lambda$ are obtained by matching the solution $\phi(y)$ in (3.57) and that obtained by the Forbenious expansion at $y=\lambda$ given in (3.38). The expansion in (3.38) is asymptotically expanded to $y \rightarrow 0$, while the $\frac{1}{\lambda}$ expansion in (3.49) is expanded to $y \rightarrow \infty$ and the function and the derivatives are matched to determine $A$ and $B$. Though we do not explicitly require $A$ and $B$ as functions of $\lambda$, we can argue that

$$
B(\lambda) \rightarrow 0, \quad A(\lambda) \rightarrow \frac{1}{2}, \quad y \rightarrow \infty .
$$

The reason for this is that when $\lambda$ strictly $\infty$, the equation (3.39) reduces to that of the zero temperature case as we have observed earlier. In this situation the solution which is finite at $y \rightarrow 0$ is given by $\frac{1}{2} y^{2} K_{2}(y)^{3}$. Thus we arrive at (3.58).

Now let us study the implications of the properties of the solutions of the differential equation (3.37) have on the retarded Green's function $g_{R}(\omega)$. In terms of the scaled variables it is given by

$$
g_{R}(\lambda)=-\frac{r_{+}^{4}}{L^{2}} \lim _{y \rightarrow 0} \frac{\lambda^{4}}{y^{3}} \frac{A(\lambda) \phi^{(1)^{\prime}}(y)+B(\lambda) \phi^{(2)^{\prime}}(y)}{A(\lambda) \phi^{(1)}(y)+B(\lambda) \phi^{(2)}(y)} .
$$

Now from the behaviour of the solutions as $y \rightarrow 0$ given in (3.45), (3.48), (3.51), (3.54) and the discussions below (3.49), (3.55) we see that the $y \rightarrow 0$ limit in (3.59) reduces to

$$
\begin{aligned}
g_{R}(\lambda) & =-\frac{r_{+}^{4}}{L^{2}} \lim _{y \rightarrow 0}\left(\frac{1}{y^{3}}\left(\lambda^{4} g_{0}^{(1)}(y)+g_{1}^{(1)}\right)+\frac{1}{4} \frac{B(\lambda)}{A(\lambda)}\right) \\
& =-\frac{r_{+}^{4}}{L^{2}}\left(\lim _{y \rightarrow 0} \frac{1}{y^{3}} \lambda^{4} g_{0}^{(1)}(y)-\frac{6}{5}+\frac{1}{4} \frac{B(\lambda)}{A(\lambda)}\right) .
\end{aligned}
$$

In the second line of the above equations we have substituted the expansion of $g_{1}^{(1)}(y)$ near the origin which is given in (3.45). An important point to note is that the above

\footnotetext{
${ }^{3}$ The $\frac{1}{2}$ is a normalization such that the solution $\frac{1}{2} y^{2} K_{2}(y) \rightarrow 1$ as $y \rightarrow 0$.
} 
result is true for all values of $\lambda$ or frequency. We can further take the limit $\lambda \rightarrow \infty$ and using (3.58) we are left with

$$
\lim _{\lambda \rightarrow \infty} g_{R}(\lambda)=-\frac{r_{+}^{4}}{L^{2}}\left(\lim _{y \rightarrow 0} \frac{1}{y^{3}} \lambda^{4} g_{0}^{(1)}-\frac{6}{5}\right) .
$$

As we have observed the leading contribution $g_{0}^{(1)}$ is identical to the zero temperature case, it is divergent as $\lambda \rightarrow \infty$. From the expression of $g_{0}^{(1)}$ in (3.43) we see that this term also has $1 / y^{2}$ and $\log (y)$ divergences as $y \rightarrow 0$ and needs to be regulated with appropriate counter terms. The next order correction in this limit is the finite contribution from the constant piece involving $g_{1}$. Thus the behaviour of the Green's function does not satisfy property 2 .

To regulate the Green's function so that it satisfies property 2 we follow [8] and consider

$$
\delta G_{R}(\omega)=G_{R}(\omega, T)-G_{R}(\omega, 0)+\frac{N^{2}}{8 \pi^{2} L^{6}} \frac{r_{+}^{4}}{L^{2}} \frac{6}{5}+P .
$$

Let us recall the definition of each of the terms in the above expression.

$$
G_{R}(\omega, T)=-\frac{N^{2}}{8 \pi^{2} L^{6}} g_{R}(\omega)-P+G_{\text {counter }}(\omega, T)
$$

Here we have just substituted the expressions for $\hat{G}_{R}(\omega)$ and $G_{\text {contact }}$ in the expression for the Green's function given in (3.8). The $T=0$ Green's function is given by

$$
\begin{array}{r}
G_{R}(\omega, 0)=\frac{N^{2}}{8 \pi^{2} L^{6}} \frac{r_{+}^{4}}{L^{2}} \lim _{y \rightarrow 0} \frac{1}{y^{3}} \lambda^{4} g_{0}^{(1)}(y)+G_{\text {counter }}(\omega), \\
=\frac{N^{2}}{8 \pi^{2} L^{4}} \omega^{4} \lim _{y \rightarrow 0} \frac{g_{0}^{(1)}(y)}{y^{3}}+G_{\text {counter }}(\omega, 0) .
\end{array}
$$

Note that there is no contact term in this case since at $T=0$, the pressure vanishes. We now point out that we have the following equality

$$
G_{\text {counter }}(\omega, 0)=G_{\text {counter }}(\omega, T) \text {. }
$$

The reason is clear because the $r^{2}$ and $\log (r)$ divergences occur only in the term $g_{0}^{(1)}$ and this term is common both for $T \neq 0$ and $T=0$. Thus the counter term to cancel these divergences must be identical. We also can infer that they are proportional to $\omega^{4}$. Now let us examine the behaviour of $\delta G_{R}(\omega)$ as $\omega \rightarrow \infty$. Substituting all the equations we see that

$$
\lim _{\omega \rightarrow \infty} \delta G_{R}(\omega)=\lim _{\lambda \rightarrow \infty} \frac{N^{2}}{8 \pi^{2} L^{6}} \frac{r_{+}^{4}}{4 L^{2}} \frac{B(\lambda)}{A(\lambda)} \rightarrow 0 .
$$

Thus $\delta G_{R}(\omega)$ satisfies the property 2. What has been essentially done by the construction in (3.62) is the divergent and constant pieces of the Green's function as 
$\omega \rightarrow \infty$ are subtracted out. Now that $\delta G_{R}(\omega)$ satisfies property 2. we can apply Cauchy's theorem and obtain the sum rule. For later purposes note that

$$
\begin{aligned}
\operatorname{Im} \delta G_{R}(\omega) & =\operatorname{Im} G_{R}(\omega, T)-\operatorname{Im} G_{R}((\omega, 0), \\
& =\rho(\omega, T)-\rho(\omega, 0),
\end{aligned}
$$

since the remaining expressions in (3.62) are real.

\subsection{The sum rule}

Now that property 1 as well as property 2 are satisfied by the function $\delta G_{R}(\omega)$, we can evaluate the LHS of the sum rule. This is given by

$$
\delta G_{R}(0)=G_{R}(0, T)-G_{R}(0,0)+\frac{N^{2}}{8 \pi^{2} L^{6}} \frac{r_{+}^{4}}{L^{2}} \frac{6}{5}+P .
$$

Now we also have

$$
\begin{aligned}
G_{R}(0, T) & =-\frac{N^{2}}{8 \pi^{2} L^{6}} g_{R}(0)-P, \\
& =-P+G_{\text {counter }}(0, T), \\
G_{R}(0,0) & =0
\end{aligned}
$$

Here we have used that fact that $g_{R}(0)=0$ and the fact that $G_{\text {counter vanishes at }}$ $\omega=0$ since as we have seen earlier that they are proportional to $\omega^{4}$. Using the equations (3.69) in (3.68) we obtain

$$
\delta G_{R}(0)=\frac{N^{2}}{8 \pi^{2} L^{6}} \frac{r_{+}^{4}}{L^{2}} \frac{6}{5}=\frac{2}{5} \epsilon
$$

Now using (3.67) and the fact that $\delta G_{R}(\omega)$ satisfies both property 1 and property 2 we obtain the shear sum rule derived in [8]

$$
\frac{2}{5} \epsilon=\frac{1}{\pi} \int_{-\infty}^{\infty} \frac{d \omega}{\omega}\left(\rho(\omega)-\rho_{T=0}(\omega)\right) .
$$

Note that the LHS of the sum rule essentially originates from the constant term in the function $g_{R}(\omega)$ as $\omega \rightarrow \infty$.

\subsection{Sum rule from OPE}

From the explicit derivation of the sum rule, we see that the LHS side essentially depends on the high frequency behaviour of the Green's function. In fact it is just proportional to the constant term in the high energy behaviour of the Green's function. Coefficients in the Operator product expansion of the stress tensor contains the information of the short distance or high energy behaviour of the Green's function. 
Thus we should be able to obtain the sum rule from the OPE of the stress tensor [B]. This OPE is given by

$$
T_{\mu \nu}(x) T_{\rho \sigma}(0) \sim C_{T} \frac{I_{\mu \nu, \rho \sigma}}{x^{8}}+\hat{A}_{\mu \nu \rho \sigma \alpha \beta}(x) T_{\alpha \beta}(0)+\cdots .
$$

$\hat{A}$ contains various Lorentz structures which can be found in [16]. The important property of them which is seen form conformal invariance is that they all scales like $1 / x^{4}$. Now we take the Fourier transform of the above OPE and set $q=0$ and $\omega \rightarrow \infty$. By a simple scaling analysis it can be seen that the term proportional to $\frac{C_{T}}{x^{8}}$ scales like $\omega^{4}$. This divergence is the same divergence seen in the gravity calculation due to the terms which are identical to the zero temperature case. The term proportional to $\hat{A}$ gives rise to a constant independent of $\omega$. Thus the constant contribution to the Green's function as $\omega \rightarrow \infty$ arises due to the one point function of the stress tensor in the thermal ensemble. Now evaluating

$$
\lim _{\omega \rightarrow \infty}\left(G_{R}(\omega, T)-G_{R}(\omega, 0)\right)=-P-\frac{2}{5} \epsilon .
$$

We have used the result obtained in [8] but in the Minkowski space and the convention of [11]. From hydrodynamics since the Green's function is defined as the response of the changes in the stress tensor on perturbing the metric, the zero frequency value of the Green's function is just given by negative of the pressure.

$$
G_{R}(0, T)=-P, \quad G_{R}(\omega, 0)=0 .
$$

On defining

$$
\delta G_{R}(\omega T)=G_{R}(\omega, T)-G_{R}(\omega, 0)+P+\frac{2}{5} \epsilon,
$$

we see that the LHS side of the sum rule is just the difference between the constant terms in the high frequency behaviour and the zero frequency behaviour of the Green's function. This results in the sum rule given in (3.71).

\section{The shear sum rule for the R-charged D3-brane}

In this section we examine the gravity dual of $\mathcal{N}=4$ Yang-Mills at finite chemical potential and finite temperature and re-derive the sum rule. Since there are 3 R-charges corresponding to the Cartan's of $S O(6)$, it is possible to turn on 3 independent chemical potentials. The gravity dual of this system is given by the R-charged black hole of Behrndt, Cvetic and Sabra [17]. Using the differential equation of the massless minimally coupled scalar in this background, we obtain the retarded Green's function of the $T_{x y}$ component of the stress tensor. Examining this differential equation and the method discussed for the uncharged D3-brane, we will show that the 
regulated Green's function satisfies both property 1 and property 2 which is necessary for the derivation of the sum rule. We show that the sum rule is corrected by terms which depend on the 3 chemical potentials. We explain these additional terms in the LHS of the sum rule due to the presence of scalars in the OPE of the stress tensor. The procedure to demonstrate property 1 and property 2 of the retarded Green's function parallels that of the uncharged D3-brane, therefore we will be brief and highlight only the differences.

\subsection{Green's function from gravity}

We begin with the metric for the R-charged D3-brane with all the three charges turned on which is given by

$$
\begin{aligned}
d s_{5}^{2} & =-\mathcal{H}^{-2 / 3} \frac{\left(\pi T_{0} L\right)^{2}}{u} f d t^{2}+\mathcal{H}^{1 / 3} \frac{\left(\pi T_{0} L\right)^{2}}{u}\left(d x^{2}+d y^{2}+d z^{2}\right)+\mathcal{H}^{1 / 3} \frac{L^{2}}{4 f u^{2}} d u^{2} \\
f(u) & =\mathcal{H}(u)-u^{2} \prod_{i=1}^{3}\left(1+k_{i}\right), \quad H_{i}=1+k_{i} u, \quad k_{i} \equiv \frac{q_{i}}{r_{H}^{2}}, \quad T_{0}=\frac{r_{+}}{\pi L^{2}}, \\
u & =\frac{r_{+}^{2}}{r^{2}}, \quad \mathcal{H}=\left(1+k_{1} u\right)\left(1+k_{2} u\right)\left(1+k_{3} u\right) .
\end{aligned}
$$

The scalar fields and the gauge fields in this background are given by

$$
X^{i}=\frac{\mathcal{H}^{1 / 3}}{H_{i}(u)}, \quad A_{t}^{i}=\frac{\tilde{k}_{i} \sqrt{2} u}{L H_{i}(u)}, \quad \tilde{k}_{i}=\sqrt{q_{i}} L \prod_{i=1}^{3}\left(1+k_{i}\right)^{1 / 2}
$$

The above metric is a solution of the equations of motion of the STU-model given by action

$$
\begin{aligned}
& S=\frac{N^{2}}{8 \pi^{2} L^{3}} \int d^{5} x \sqrt{-g} \mathcal{L}, \\
& \mathcal{L}=R+\frac{2}{L^{2}} \mathcal{V}-\frac{1}{2} G_{i j} F_{\mu \nu}^{i} F^{\mu \nu j}-G_{i j} \partial_{\mu} X^{i} \partial^{\mu} X^{j}+\frac{1}{24 \sqrt{-g}} \epsilon^{\mu \nu \rho \sigma \lambda} \epsilon_{i j k} F_{\mu \nu}^{i} F^{\rho \sigma j} A_{\lambda}^{k},
\end{aligned}
$$

where $F_{\mu \nu}^{i}, i=1,2,3$ are the field-strengths for the three Abelian gauge fields. The three scalar fields $X^{i}$ 's are subject to the constraint $X^{1} X^{2} X^{3}=1$. The metric on the scalar manifold is given by

$$
G_{i j}=\frac{1}{2} \operatorname{diag}\left\{\left(X^{1}\right)^{-2},\left(X^{2}\right)^{-2},\left(X^{3}\right)^{-2}\right\} .
$$

The scalar potential is given by

$$
\mathcal{V}=2\left(\frac{1}{X^{1}}+\frac{1}{X^{2}}+\frac{1}{X^{3}}\right)
$$


It is useful to recall the following thermodynamic data of this black hole [18]. The Hawking temperature $T_{H}$, entropy density $s$, energy density $\epsilon$, pressure $P$, charge densities $\rho_{i}$ and the conjugate chemical potentials $\mu_{i}$ are given by

$$
\begin{aligned}
T_{H}=\frac{2+k_{1}+k_{2}+k_{3}-k_{1} k_{2} k_{3}}{2 \sqrt{\left(1+k_{1}\right)\left(1+k_{2}\right)\left(1+k_{3}\right)}} T_{0}, & s=\frac{\pi^{2} N^{2} T_{0}^{3}}{2} \prod_{i=1}^{3}\left(1+k_{i}\right)^{1 / 2}, \\
\epsilon=\frac{3 \pi^{2} N^{2} T_{0}^{4}}{8} \prod_{i=1}^{3}\left(1+k_{i}\right), & P=\frac{\pi^{2} N^{2} T_{0}^{4}}{8} \prod_{i=1}^{3}\left(1+k_{i}\right), \\
\rho_{i}=\frac{\pi N^{2} T_{0}^{3}}{8} \sqrt{2 k_{i}} \prod_{i=1}^{3}\left(1+k_{i}\right), & \mu_{i}=\frac{\pi T_{0} \sqrt{2 k_{i}}}{\left(1+k_{i}\right)} \prod_{i=1}^{3}\left(1+k_{i}\right) .
\end{aligned}
$$

The thermodynamical stability condition of this black hole is given by

$$
2-k_{1}-k_{2}-k_{3}+k_{1} k_{2} k_{3}>0 .
$$

As before, to study the retarded correlator $G_{R}(\omega, T)$ we must examine the equation of motion of a massless minimal coupled scalar in this background. We consider the perturbation

$$
\delta g_{x y}=\phi(r) \mathcal{H}^{1 / 3} \frac{r^{2}}{L^{2}} e^{-i \omega t},
$$

whose equations of motion in radial co-ordinate $r$ is given by [18]

$$
\partial_{r}^{2} \phi+\left(\frac{F^{\prime}}{F}+\frac{3}{r}\right) \partial_{r} \phi+\frac{\mathcal{H} \omega^{2}}{F^{2}} \phi=0,
$$

where again $F$ is given by

$$
F=\frac{r^{2}}{L^{2}} f
$$

and $f$ is defined in (4.1). This equation is similar to the uncharged case except for the presence of $\mathcal{H}$ as coefficient of $\omega^{2}$ and the fact that $f$ depends on the constants $k_{i}$. To obtain the retarded Green's function, we must impose in-going boundary conditions at the horizon $r_{+}$and obtain $\phi$ at the boundary $r \rightarrow \infty$. Then the Green's function is given by the expression in (3.8). $G_{\text {contact }}$ is the contribution of the contact terms obtained from the on shell effective action. For the charged case, this can be evaluated by using following action

$$
S=\frac{N^{2}}{8 \pi^{2} L^{3}} \int d^{5} x \sqrt{-g} \mathcal{L}+\frac{N^{2}}{4 \pi^{2} L^{3}} \int_{\partial M_{5}} d^{4} x \sqrt{-h} K+\frac{N^{2}}{4 \pi^{2} L^{3}} \int_{\partial M_{5}} \sqrt{-h} W .
$$

Here the second term is the Gibbons-Hawking boundary term while the third term is the term required to make the action finite in the limit $r \rightarrow \infty$ [19]. It is given by

$$
W=-\frac{\mathcal{H}^{1 / 3}}{L} \sum_{i=1}^{3} H_{i}^{-1}
$$


Using this, it can be shown that the contact terms are again obtained from the on shell action given in (3.9) [18 ${ }^{4}$. Therefore we obtain

$$
G_{\text {contact }}=-P
$$

There is an alternative indirect method to infer that the contact term is given by $-P$. Contact terms do not depend on frequency, therefore one can obtain them by taking the zero frequency limit. It will be shown that $\hat{G}(\omega, T) \rightarrow 0$ in the $\omega \rightarrow 0$ limit, further more the $G_{\text {counter }}$ as in the case of the uncharged case behave as $\omega^{4}$. Thus in the zero frequency limit the only contribution to the Green's function is from $G_{\text {contact }}$. Now from hydrodynamics, the Greens' function is the response of the stress tensor $T_{x y}$ to the metric fluctuation $\delta g_{x y}$. At zero frequency, this is just $-P$. Thus we have $G_{\text {contact }}=-P$.

Before we begin our analysis, we will need the behaviour of the solutions near the horizon and the boundary. The behaviour of the solutions near the horizon is given by

$$
\phi(r) \sim\left(r-r_{+}\right)^{ \pm i \alpha \omega}
$$

where $\alpha$ can be written as

$$
\begin{aligned}
\alpha & =\frac{1}{F_{h}} \sqrt{\left(1+k_{1}\right)\left(1+k_{2}\right)\left(1+k_{3}\right)} \\
F & =\left(r-r_{h}\right) F_{h}+\cdots, \quad F_{h}=\frac{2 r_{+}}{L^{2}}\left(2+k_{1}+k_{2}+k_{3}-k_{1} k_{2} k_{3}\right) .
\end{aligned}
$$

As $r \rightarrow \infty$, the metric asymptotes to $A d S_{5}$, therefore the behaviour of $\phi(r)$ is the same as in the uncharged case and is given by (3.14). Finally the equations for $\phi(r)$ can be obtained by variation of the following action

$$
S_{\phi}=\int_{r_{h}}^{\infty} d r F r^{3}\left(\left|\phi^{\prime}(r)\right|^{2}-\frac{\omega^{2} \mathcal{H}}{F^{2}}|\phi(r)|^{2}\right) .
$$

The only change in this action compared to the uncharged case in (3.15) is the presence of $\mathcal{H}$ in the term proportional to the frequency.

\subsection{Green's function in the $\omega$-plane}

In this subsection we will discuss the analytic properties of the function

$$
g_{R}(\omega)=\lim _{r \rightarrow \infty} \frac{F r^{3} \phi^{\prime}}{\phi}
$$

in the complex $\omega$ plane. The discussion closely follows that of the uncharged case. The difference arises mainly in the behaviour of the $\omega \rightarrow \infty$ limit which we will highlight.

\footnotetext{
${ }^{4}$ See equation 4.24 of 18
} 


\section{No poles for $\operatorname{Im} \omega>0$}

Here the discussion is identical to that of the uncharged case. Poles or divergences in $g_{R}(\omega)$ correspond to quasi-normal modes of the equation (3.6). Quasi-normal modes are solutions to the equation (3.6) with the following boundary conditions

$$
\begin{aligned}
\phi(r) \sim\left(r-r_{+}\right) e^{-i \alpha \omega}, & r \rightarrow r_{+}, \\
\phi(r) \sim r^{-4}, & r \rightarrow \infty .
\end{aligned}
$$

One can follow the same arguments as in the case of the uncharged situation to show that such modes do not exist for $\operatorname{Im} \omega>0$. We need to employ the action given in (4.16) for this purpose. The fact that $\mathcal{H}>0$ in the domain $r_{+}<r<\infty$ also ensures that the action $S_{\phi}$ evaluated on the quasi-normal mode is positive definite. This enables us to repeat the same arguments as in the case of zero chemical potential.

\section{No poles of $\omega$ real and $\omega \neq 0$}

Here again the same reasoning developed for the uncharged case earlier using the Wronskian, ensures that quasi-normal modes do not exist on the real line $\omega \neq 0$. This implies that there are no poles or divergences in $g_{R}(\omega)$ in this domain.

\section{No poles for $\omega=0$}

The behaviour of the function $g_{R}$ as $\omega \rightarrow 0$ can be determined from the differential equation (4.9) as follows. We define

$$
\tilde{g}(r)=\frac{\phi^{\prime}}{\omega \phi(r)} .
$$

Using the equation (4.9) we find the $\tilde{g}(r)$ satisfies the equation

$$
\tilde{g}^{\prime}+\omega \tilde{g}^{2}+\left(\frac{F^{\prime}}{F}+\frac{3}{r}\right) \tilde{g}(r)+\frac{\omega \mathcal{H}}{F^{2}}=0 .
$$

The ingoing boundary condition implies that $\tilde{g}$ satisfies

$$
\tilde{g} \rightarrow \frac{-i \sqrt{\left(1+k_{1}\right)\left(1+k_{2}\right)\left(1+k_{3}\right)}}{F_{h}\left(r-r_{+}\right)}, \quad r \rightarrow r_{+} .
$$

The solution of (4.20) in the $\omega \rightarrow 0$ limit which satisfies the above boundary condition is given by

$$
\tilde{g}=-i \frac{r_{+}^{3} \sqrt{\left(1+k_{1}\right)\left(1+k_{2}\right)\left(1+k_{3}\right)}}{r^{3} F}, \quad \omega \rightarrow 0 .
$$

This implies that

$$
\lim _{\omega \rightarrow 0} g_{R}(\omega)=\omega \lim _{r \rightarrow \infty} r^{3} F \tilde{g}=-i \omega r_{+}^{3} \sqrt{\left(1+k_{1}\right)\left(1+k_{2}\right)\left(1+k_{3}\right)} .
$$


Thus $g_{R}(\omega)$ is proportional to $\omega$ as $\omega \rightarrow 0$. Examining the equation (4.20), it can be seen that it admits a power series expansion in $\omega$ around the origin.

No zeros for $\operatorname{Im} \omega>0, \omega \neq 0$

As argued for the uncharged case, zeros or points at which the Green's function vanish correspond to the boundary condition

$$
\begin{aligned}
\phi(r) & \sim\left(r-r_{+}\right)^{-i \alpha \omega}, \quad r \rightarrow r_{+}, \\
r^{3} F \phi^{\prime}(r) \rightarrow 0, \quad r \rightarrow \infty . &
\end{aligned}
$$

Using the same reasoning discussed for the uncharged situation, it is easy to see that there are no modes which have the above boundary condition for $\operatorname{Im} \omega>0, \omega \neq 0$. This implies that there are no zeros or points at which the Green's function vanishes in this domain.

No branch cuts for $\operatorname{Im} \omega \geq 0$

From the discussion above, we have seen that the Green's function does not have any singularities, nor does it vanish any where in the upper half plane except at the origin. At the origin it admits an analytic power series expansion. As we have argued for the uncharged D3-brane case the general theorem in ([15]) ensures that the local Forbenius expansion of the solution is smooth with respect to the parameter $\omega$ since the differential equation and the boundary condition are both smooth with respect to this parameter $\omega$. Now from (4.17) it is easy to see that the $n^{\text {th }}$ order derivatives of the Green's function with respect to the parameter $\omega$ exist. This is because the singularities for the $n^{\text {th }}$ order derivative can arise only from the zeros of $\phi$ which are its quasi-normal modes. We have already shown that there are no quasi-normal modes in the upper half-plane, hence we conclude that arbitrary derivatives of the Green's function with respect to $\omega$ exists in the upper half plane. Thus the Green's function does not have any branch cuts in the upper half-plane

Therefore the expansion at $\omega=0$ can be extended to the entire upper half plane and the function is analytic in the upper half plane. Thus the Green's function satisfies the property 1 for the derivation of the sum rule.

\section{Behaviour as $\omega \rightarrow \infty$}

To obtain the asymptotic behaviour of the Green's function it is again convenient to work in the scaled variables

$$
z=\frac{r_{+}}{r}, \quad i \lambda=\frac{L^{2}}{r_{+}} \omega .
$$


We then expand the equation (4.9) in powers of $\frac{1}{\lambda}$. For this it is convenient to express the background metric given in (4.1) in Fefferman-Graham coordinates. The metric for the charged D3-brane in Fefferman-Graham coordinates is given by

$$
d s^{2}=\frac{L^{2}}{z^{2}}\left(\left(-1+\sum_{i=2}^{\infty} a_{i}\left(z^{2}\right)^{i}\right) d t^{2}+\left(1+\sum_{i=2}^{\infty} b_{i}\left(z^{2}\right)^{i}\right)\left(d \vec{x}^{2}\right)+d z^{2}\right) .
$$

The details of the transformation to these co-ordinates are given in the appendix. Note that in this expansion the term $a_{1}, b_{1}$ is missing. Here the boundary is at $z=0$. It will be seen that the coefficients $a_{2}, b_{2}$ are crucial to determine the asymptotic behaviour of the Green's function. These are evaluated in (A.5). The equation for the minimally coupled scalar in this co-ordinates is given by

$$
\phi^{\prime \prime}-\frac{1}{z}\left(3+\sum_{i=2}^{\infty} \tilde{a}_{i}\left(z^{2}\right)^{i}\right) \phi^{\prime}-\omega^{2}\left(1+\sum_{i=2}^{\infty} \tilde{b}_{i}\left(z^{2}\right)^{i}\right) \phi=0,
$$

where $\tilde{a}_{i}, \tilde{b}_{i}$ are related to the coefficients $a_{i}, b_{i}$. Again the leading coefficients are important for our later use which are related by

$$
\tilde{a}_{2}=2\left(a_{2}-3 b_{2}\right), \quad \tilde{b}_{2}=a_{2} .
$$

We now rescale the co-ordinate $z$ by defining

$$
y=\lambda z .
$$

This leads to the equation for the minimally coupled scalar as a $\frac{1}{\lambda}$ expansion.

$$
\phi^{\prime \prime}(y)-\frac{1}{y}\left(3+\sum_{i=2}^{\infty} \tilde{a}_{i}\left(\frac{y^{2}}{\lambda^{2}}\right)^{i}\right) \phi^{\prime}(y)-\left(1+\sum_{i=2}^{\infty} \tilde{b}_{i}\left(\frac{y^{2}}{\lambda^{2}}\right)^{i}\right) \phi(y)=0 .
$$

Now this expansion is similar to that obtained for the un-charged situation given in (3.49). We can therefore use the same method to solve it order by order in $1 / \lambda$. We define

$$
g(y)=\frac{\phi^{\prime}(y)}{\phi(y)} .
$$

From the equation given in (4.30) we see that the $g$ admits an expansion of the form

$$
g=g_{0}+\frac{1}{\lambda^{4}} g_{1}+\frac{1}{\lambda^{6}} g_{2}+\cdots .
$$

The equations satisfied by these can be obtain from (4.30) and the first few orders are given by

$$
\begin{aligned}
& g_{0}^{\prime}+g_{0}^{2}-\frac{3}{y}-1=0, \\
& g_{1}^{\prime}+\left(2 g_{0}-\frac{3}{y}\right) g_{1}-\tilde{a}_{2} y^{3} g_{0}-\tilde{b}_{2} y^{4}=0, \\
& g_{2}^{\prime}+\left(2 g_{0}-\frac{3}{y}\right) g_{2}-\tilde{a}_{3} y^{5} g_{0}-\tilde{b}_{3} y^{6}=0 .
\end{aligned}
$$


We will now follow the same procedure as done for the uncharged case to obtain two independent solutions to (4.30). The zeroth order solution is same as the uncharged case since in the $\lambda \rightarrow \infty$ limit, the equation (4.30) reduces to the $\lambda \rightarrow \infty$ limit of (3.49). This is expected since asymptotically the metric (4.1) reduces to $A d S_{5}$. Therefore we have

$$
g_{0}^{(1)}=-\frac{K_{1}(y)}{K_{2}(y)}=\frac{d}{d y}\left(\log \left(y^{2} K_{2}(y)\right)\right), \quad g_{0}^{(2)}=\frac{I_{1}(y)}{I_{2}(y)}=\frac{d}{d y}\left(\log \left(y^{2} I_{2}(y)\right)\right) .
$$

Let us first find the $\frac{1}{\lambda}$ expansion around the solution $g_{0}^{(1)}$. The first order correction is given by

$$
g_{1}^{(1)}(y)=\frac{1}{y K_{2}^{2}(y)} \int_{0}^{y} d y\left(\tilde{a}_{2} y^{3} g_{0}+\tilde{b}_{2} y^{4}\right) y K_{2}^{2}(y)+\frac{c_{1}}{y K_{2}^{2}(y)} .
$$

We set $c_{1}=0$, so as not to change the asymptotics of the solution at $y \rightarrow \infty$. On performing the integral we obtain

$$
\begin{aligned}
g_{1}^{(1)}(y) & =\frac{\tilde{a}_{2}}{2} y^{3}+\frac{\tilde{b}_{2}}{10} y^{5}\left(1-\frac{K_{3}^{2}}{K_{2}^{2}}\right) \\
& =\left(\frac{\tilde{a}_{2}}{2}-\frac{8}{5} \tilde{b}_{2}\right) y^{3}-\frac{3}{10} \tilde{b}_{2} y^{5}+O\left(y^{7}, y^{7} \log (y)\right)
\end{aligned}
$$

Similarly, the solution to $g_{2}$ is given by

$$
g_{2}^{(1)}(y)=\frac{1}{y K_{2}^{2}(y)} \int_{0}^{y}\left(\tilde{a}_{3} y^{5} g_{0}+\tilde{b}_{3} y^{6}\right) y K_{2}^{2}(y) .
$$

From the expansions of the functions involved, one sees that

$$
g_{2}^{(1)}(y)=y^{7}+O\left(y^{9}, y^{9} \log y\right)
$$

Similarly from the structure of the functions in the $\frac{1}{\lambda}$ expansion, it is easy to see that

$$
g_{n}(y) \sim y^{m}, \quad m \geq 7, \quad \text { for } n \geq 2, \quad y \rightarrow 0
$$

Using this procedure it is possible to construct all the $g_{n}^{(1)}$ 's. Form this we find that the first solution of the differential equation is given by

$$
\begin{aligned}
\phi^{(1)}(y) & =\exp \left(\int_{0}^{y} d y\left(g_{0}+\frac{g_{1}}{\lambda^{4}}+\frac{g_{2}}{\lambda^{6}}+\cdots\right)\right. \\
& =y^{2} K_{2}(y)\left(1+\frac{1}{\lambda^{4}} \int_{0}^{y} d y g_{1}(y)+\frac{1}{\lambda^{6}} \int_{0}^{y} d y g_{2}(y)+\cdots\right) .
\end{aligned}
$$

Note that

$$
\phi^{(1)} \sim \text { constant }, \quad y \rightarrow 0 .
$$


From $(4.38)$ we see that $\frac{1}{\lambda^{4}}$ term in (4.42) goes as $y^{4}$ near the origin. The higher order terms are further suppressed as $y \rightarrow 0$. A similar construction can be done starting with the seed $g_{0}^{(2)}$. The first order term about this seed is

$$
\begin{aligned}
g_{1}^{(2)}(y) & =\frac{1}{y I_{2}^{2}(y)} \int_{0}^{y} d y\left(\tilde{a}_{2} y^{3} g_{0}+\tilde{b}_{2} y^{4}\right), \\
& =\frac{\tilde{a}_{2}}{2} y^{3}+\frac{\tilde{b}_{2}}{10} y^{5}\left(1-\frac{I_{3}^{2}}{I_{2}^{2}}\right) .
\end{aligned}
$$

Similarly $g_{2}^{(2)}(y)$ can be written as

$$
g_{2}^{(2)}(y)=\frac{1}{y I_{2}^{2}(y)} \int_{0}^{y}\left(\tilde{a}_{3} y^{5} g_{0}+\tilde{b}_{3} y^{6}\right) y I_{2}^{2}(y)
$$

Examining this solution, we see that the behaviour of this function as $y \rightarrow 0$ is given by

$$
g_{2}^{(2)}(y) \sim y^{5}+O\left(y^{7}\right), \quad y \rightarrow 0 .
$$

From the general form of the equations for $g_{n}$, it can be seen that

$$
g_{n}^{(2)}(y) \sim y^{m}, \quad m \geq 5 \quad \text { for } \quad n \geq 2, \quad y \rightarrow 0 .
$$

Thus one obtains the solution

$$
\phi^{(2)}(y)=y^{2} I_{2}(y)\left(1+\frac{1}{\lambda^{4}} \int_{0}^{y} g_{1}(y)+\frac{1}{\lambda^{6}} \int_{0}^{y} g_{2}(y)+\cdots\right) .
$$

It is important to observe that

$$
\phi^{(2)}(y) \sim y^{4}, \quad y \rightarrow 0
$$

which is same as in the case of the uncharged case. Again from (4.44) and (4.47), the terms in the $\frac{1}{\lambda}$ expansion are suppressed as $y \rightarrow 0$.

Once the two independent solutions have been constructed in this manner, we can write the solutions which obey the boundary conditions as

$$
\phi(y)=A(\lambda) \phi^{(1)}(y)+B(\lambda) \phi^{(2)} .
$$

The coefficients $A, B$ depend on $\lambda$ and can be obtained by matching $\phi(y)$ and its derivative with the near solution. The near solution is obtained in terms of a Forbenius power series expansion at the horizon. The solution is of the form

$$
\phi=\left(1-\frac{y}{\lambda}\right)^{\frac{\alpha \lambda r_{+}}{L^{2}}}\left(\sum_{j=0}^{\infty} a_{j}\left(1-\frac{y}{\lambda}\right)^{j}\right) .
$$

Now though we do not need the detail dependence of the coefficient $B$ on $\lambda$, it is clear that using the same argument as in the uncharged situation we find

$$
B(\lambda) \rightarrow 0, \quad A(\lambda) \rightarrow \frac{1}{2}, \quad \text { as } \quad \lambda \rightarrow \infty
$$


The reason is that we know that at $\lambda \rightarrow \infty$, the equation reduces to that of the $T=0$ limit. In this situation the solution which is finite at $y \rightarrow \infty$ is $\frac{1}{2} y^{2} K_{2}(y)$.

With this input and using the same steps followed in the uncharged situation we find the Green's function to be

$$
g_{R}(\lambda)=-\frac{r_{+}^{4}}{L^{2}}\left(\lim _{y \rightarrow 0} \frac{\lambda^{4}}{y^{3}} g_{0}^{(1)}(y)-\frac{3}{5}\left(a_{2}+5 b_{2}\right)+\frac{1}{4} \frac{B(\lambda)}{A(\lambda)}\right) .
$$

In this we have substituted for $\tilde{a}_{2}, \tilde{b}_{2}$ from (4.28). Again, the above result is valid for all values of $\lambda$. Taking the $\lambda \rightarrow \infty$ limit we are left with

$$
\lim _{\lambda \rightarrow \infty} g_{R}(\lambda)=-\frac{r_{+}^{4}}{L^{2}}\left(\lim _{y \rightarrow 0} \frac{\lambda^{4} g_{0}^{(1)}(y)}{y^{3}}-\frac{3}{5}\left(a_{2}+5 b_{2}\right)\right),
$$

where we have used (4.52). As we have seen earlier the $\lambda^{4}$ contribution is identical to the zero temperature case. There is also a constant term due to the first order correction $g^{(1)}$. Thus the Green's function does not satisfy property 2 and needs to be regulated.

As before, to regulate the Green's function we consider

$$
\delta G_{R}(\omega)=G_{R}(\omega, T)-G_{R}(\omega, 0)+\frac{N^{2}}{8 \pi^{2} L^{6}} \frac{r_{+}^{4}}{L^{2}}\left(\frac{3}{5}\left(a_{2}+5 b_{2}\right)\right)+P .
$$

Now using all our previous results and following the same steps as in the case of the uncharged situation we have

$$
\delta G_{R}(\omega) \rightarrow 0, \quad \omega \rightarrow \infty .
$$

We have essentially subtracted the divergent and the constant term in $G_{R}(\omega)$ so that property 2 is true on $\delta G_{R}(\omega)$ which can then be used to obtain the sum rule. Note that for the charged D3-brane case the equation (3.67) still holds since all the terms subtracted to construct $\delta G_{R}(\omega)$ are still real.

\subsection{The sum rule}

We can now use Cauchy's theorem on the function $\delta G_{R}(\omega)$ and derive the sum rule. The sum rule for the charged D3-brane then is given by

$$
\delta G_{R}(0)=\int_{-\infty}^{\infty} \frac{d \omega}{\omega}\left(\rho(\omega)-\rho_{T=0}(\omega)\right)
$$

where

$$
\delta G_{R}(0)=\frac{N^{2}}{8 \pi^{2} L^{6}} \frac{r_{+}^{4}}{L^{2}}\left(\frac{3}{5}\left(a_{2}+5 b_{2}\right)\right) .
$$


We can now substitute the values of $a_{2}, b_{2}$ from (A.5). We obtain

$$
\begin{aligned}
\delta G_{R}(0)= & \frac{N^{2}}{8 \pi^{2} L^{6}} \frac{r_{+}^{4}}{L^{2}} \frac{3}{5}\left(2\left(1+k_{1}\right)\left(1+k_{2}\right)\left(1+k_{3}\right)\right. \\
& \left.-\frac{1}{9}\left\{\left(k_{1}-k_{2}\right)^{2}+\left(k_{1}-k_{3}\right)^{2}+\left(k_{2}-k_{3}\right)^{2}\right\}\right) \\
= & \frac{2}{5} \epsilon-\frac{N^{2} \pi^{2} T_{0}^{4}}{120}\left\{\left(k_{1}-k_{2}\right)^{2}+\left(k_{1}-k_{3}\right)^{2}+\left(k_{2}-k_{3}\right)^{2}\right\} .
\end{aligned}
$$

Here we have rewritten the first term in terms of the energy density using its expression given in (4.6). Note that there is a modification of the sum rule which involves the chemical potential. In the next section we will show that this modification is due to the expectation values of the scalars in the charged D3-brane background. As a simple check of this hypothesis, note that for D3-brane with equal R-charge in all the 3 Cartan directions $k_{1}=k_{2}=k_{3}$, the additional term vanishes.

\subsection{Sum rule from OPE}

We have seen that the term on the LHS of the sum rule is essential due to the high frequency behaviour of the retarded Green's function. In the uncharged situation, this high frequency behaviour can be captured by the OPE of the $T_{x y}$ component of the stress tensor. Therefore if the sum rule has to be modified there must be additional terms in the OPE which contribute at finite chemical potential. Let us now re-examine the OPE of the stress tensor which is given by

$$
T_{\mu \nu}(x) T_{\rho \sigma}(0) \sim C_{T} \frac{I_{\mu \nu, \rho \sigma}}{x^{8}}+\hat{A}_{\mu \nu \rho \sigma \alpha \beta}(x) T_{\alpha \beta}(0)+B_{\mu \nu \rho \sigma}^{a}(x) \mathcal{O}_{a}(0) .
$$

Here we have included additional terms in the OPE which arise if there are scalars denoted by $\mathcal{O}_{a}$ in the theory and the three point function $\left\langle T_{\mu \nu} T_{\rho \sigma} \mathcal{O}_{a}\right\rangle$ is non-zero. From a simple scaling analysis it can be shown that operators of dimension lower than 4 in the OPE contribute to divergences as $\omega \rightarrow \infty$. Operators of dimension greater than 4 are irrelevant and operators of dimension 4 contribute to the finite terms responsible for the sum rule. Consider an operator $\mathcal{O}_{a}$ of dimension $\Delta$ in $\mathrm{OPE}$, the structure of this term will be of the form

$$
B_{\mu \nu \rho \sigma}^{a}(x) \mathcal{O}_{a}(0)
$$

where $a$ refers both to the operator as well as tensor indices if any. By conformal invariance we have the following scaling property

$$
B_{\mu \nu \rho \sigma}^{a}(\Lambda x)=\Lambda^{\Delta-8} B_{\mu \nu \rho \sigma}^{a}(x) .
$$

Now, on taking the Fourier transform of this term we obtain

$$
\int d^{4} x e^{-i \omega t} B_{\mu \nu \rho \sigma}^{a}(x) \mathcal{O}_{a}(0)=\omega^{4-\Delta} \int d^{4} \tilde{x} e^{-i \tilde{t}} B_{\mu \nu \rho \sigma}^{a}(\tilde{x}) \mathcal{O}_{a}(0) .
$$


Here we have rescaled all coordinates by replacing $x=\frac{\tilde{x}}{\omega}$. From (4.63) we see that the terms diverge for $\Delta<4$ and are irrelevant for $\Delta>4$. The finite terms in the OPE are therefore coming from operators of dimension $\Delta=4$.

We have seen from the discussion of the uncharged D3-branes, the term proportional to the stress tensor contributes to term $\frac{2}{5} \epsilon$ in the sum rule. Thus the remaining terms in (4.59) must be due to the expectation values of certain dimension 4 operators. From the structure of the extra terms in (4.59), we see that they vanish when all the charges are equal. At this special point one can see from the solution (4.2), the scalars become trivial while the gauge field still remains non-zero. Thus it must be the case that operators dual to the scalars are responsible for the additional terms in the sum rule. To find the conformal dimensions of the operators dual to these scalars we evaluate their masses. Due to the constraint $X_{1} X_{2} X_{3}=1$, there are two independent scalars. We parametrize $X_{i}$ 's in terms of these independent scalars by

$$
\begin{aligned}
& X_{1}=\exp \left(-\frac{1}{\sqrt{6}} \phi_{1}-\frac{1}{\sqrt{2}} \phi_{2}\right), \quad X_{2}=\exp \left(-\frac{1}{\sqrt{6}} \phi_{1}+\frac{1}{\sqrt{2}} \phi_{2}\right), \\
& X_{3}=\exp \left(\frac{2}{\sqrt{6}} \phi_{1}\right) .
\end{aligned}
$$

Substituting these redefinitions in the action (4.3) and expanding the scalar potential

$$
V=\frac{4}{L^{2}} \sum_{i=1}^{3} \frac{1}{X_{i}}
$$

to quadratic order in $\phi_{i}$ it can be shown that the masses of both $\phi_{1}, \phi_{2}$ is given by

$$
m^{2} L^{2}=-4
$$

From the mass-dimension relation

$$
\Delta(\Delta-4)=m^{2} L^{2}
$$

we see that the fields $\phi_{i}$ corresponds to operators of dimension 2 in the field theory and saturate the Breitenlohner-Freedman bound. In the field theory these operators correspond to two linear combinations of the following chiral primaries

$$
\operatorname{Tr}(X \bar{X}), \quad \operatorname{Tr}(Y \bar{Y}), \quad \operatorname{Tr}(Z \bar{Z}),
$$

where $X, Y, Z$ are 3 complex fields constructed out of the 6 scalars in $\mathcal{N}=4$ YangMills. These are the three scalars which are un-charged under the Cartans of $S O(6)$. Note that the combination $\operatorname{Tr}(X \bar{X})+\operatorname{Tr}(Y \bar{Y})+\operatorname{Tr}(Z \bar{Z})$ is the Konishi scalar and therefore not a chiral primary. Let us denote the two chiral primaries dual to the field $\phi_{1}, \phi_{2}$ as $O_{1}$ and $O_{2}$ respectively. 
From the gravity background it is easy to read out the expectation values of the operators $O_{1}$ and $O_{2}$ at finite chemical potential. Since these fields saturate the Breitenlohner-Freedman bound their behaviour at the boundary is given by

$$
\phi_{i}(r) \sim L\left(\alpha_{i} \frac{\log r}{r^{2}}+\beta_{i} \frac{1}{r^{2}}+\cdots\right)
$$

Here we have introduced factors of $L$ so as to ensure that $\alpha_{i}, \beta_{i}$ have the appropriate dimensions. The boundary term for the scalars given in (4.12) ensures that $\alpha_{i}$ is set to zero at the boundary. Then the expectation value of the operator dual to $\phi_{i}$ is obtained by substituting the redefinitions (4.64) and in the background value of $X_{i}$ and reading out the value of $\frac{N^{2}}{8 \pi^{2} L^{3}} \beta_{i}$ [20]. See [21, 22] for earlier work. They are given by

$$
\begin{aligned}
& \left\langle O_{1}\right\rangle=\frac{N^{2}}{8 \pi^{2} L^{3}} \beta_{1}=\frac{N^{2}}{8 \pi^{2}} \frac{r_{+}^{2}}{L^{4}} \frac{k_{1}+k_{2}-2 k_{3}}{\sqrt{6}}, \\
& \left\langle O_{2}\right\rangle=\frac{N^{2}}{8 \pi^{2} L^{3}} \beta_{2}=\frac{N^{2}}{8 \pi^{2}} \frac{r_{+}^{2}}{L^{4}} \frac{k_{1}-k_{2}}{\sqrt{2}} .
\end{aligned}
$$

Note that these expectation values have mass dimension 2 since the operators $O_{1}, O_{2}$ have mass dimension 2. They are also constant in space time.

A natural candidate for the operators $\mathcal{O}^{i}$ of dimension 4 which occur in the OPE and which can contribute to the sum rule are the squares of the operators ${ }^{5}$

$$
\mathcal{O}_{1}=\frac{1}{N} O_{1}^{2}, \quad \mathcal{O}_{2}=\frac{1}{N} O_{2}^{2}, \quad \mathcal{O}_{3}=\frac{1}{N} O_{1} O_{2}
$$

We have defined these operators with the factor of $\frac{1}{N}$ so that the two point function of all these operators are normalized as $N^{2}$.

We first show that the OPE of the stress tensor can give rise to these operators. The operators $\mathrm{O}_{1}$ and $\mathrm{O}_{2}$ are chiral primaries, and they will contain say the operator $\operatorname{Tr}(Z \bar{Z})$. Thus the operator $\mathcal{O}_{1}$ and $\mathcal{O}_{2}$ will contain the operator

$$
\mathcal{P}=\frac{1}{N}(\operatorname{Tr}(Z \bar{Z}))^{2}
$$

Therefore we evaluate the following three point function in the free field limit

$$
\left\langle T_{x y}\left(x^{1}\right) T_{x y}\left(x^{2}\right) \mathcal{P}\left(x^{3}\right)\right\rangle=\frac{1}{N^{3}}\left\langle\operatorname{Tr}\left(\partial_{x^{1}} Z \partial_{y^{1}} \bar{Z}\right) \operatorname{Tr}\left(\partial_{x^{2}} Z \partial_{x^{2}} \bar{Z}^{2}\right) \operatorname{Tr}(Z \bar{Z}) \operatorname{Tr}(Z \bar{Z})\right\rangle(4
$$

To obtain this equality we have written out only the terms which contribute to the correlator in the free field limit. We have also ignored numerical proportionality

\footnotetext{
${ }^{5}$ The operators $O_{i}$ do not occur directly in the OPE since the 3 point function $\left\langle T_{\mu \nu} T_{\rho \sigma} O_{i}\right\rangle$ vanishes in gravity.
} 
constants. Note that the two point function of the stress tensor is normalized to unity. By applying Wick's contractions we obtain

$$
\left\langle T_{x y}\left(x^{1}\right) T_{x y}\left(x^{2}\right) \mathcal{P}\left(x^{3}\right)\right\rangle=N \frac{\left(x^{1}-x^{3}\right)\left(y^{1}-y^{3}\right)\left(x^{2}-x^{3}\right)\left(y^{2}-y^{3}\right)}{\left|x^{1}-x^{3}\right|^{8}\left|x^{2}-x^{3}\right|^{8}}
$$

Therefore from the free field calculation it is evident that

$$
\left\langle T_{x y} T_{x y} \mathcal{P}\right\rangle \neq 0
$$

Any operator $\mathcal{O}$ whose three point function $\langle T T \mathcal{O}\rangle$ is non-zero will appear in the OPE of the stress tensor. In fact they will be more relevant than the stress tensor if the operators have dimension $\Delta<4$, and as relevant if the operator has dimension $\Delta=4$, see [16] below equation (6.47). Thus even in the free field limit the operators considered can arise in the OPE of the stress tensor.

Now we will show that the expectation values of the operators $\mathcal{O}_{1}$ and $\mathcal{O}_{2}$ is responsible for the modification of the sum rule. For this we evaluate the following

$$
\begin{aligned}
\left\langle\mathcal{O}_{1}\right\rangle+\left\langle\mathcal{O}_{2}\right\rangle & =\frac{1}{N}\left(\left\langle O_{1}\right\rangle^{2}+\left\langle O_{2}\right\rangle^{2}\right) \\
& =\frac{N^{3} T_{0}^{4}}{192}\left\{\left(k_{1}-k_{2}\right)^{2}+\left(k_{1}-k_{3}\right)^{2}+\left(k_{2}-k_{3}\right)^{2}\right\} .
\end{aligned}
$$

The first equality is due to large $N$ factorization. In the second line we have substituted the expectation values form (4.70) and re-expressed $r_{+}$in terms of $T_{0}$. Note that the final line of (4.76) is proportional to the additional term in the sum rule (4.59) with the exception of the factor of $N$. This fact is because the structure constant $B_{\mu \nu \rho}^{a}$ for these operators scales as $\frac{1}{N}$ in the large $N$ limit as can be seen from the normalization of our operators. This additional factor of $\frac{1}{N}$ together with the $N^{3}$ in (4.76) gives the required $N^{2}$ scaling seen in the sum rule. Therefore we conclude these additional terms in the shear sum rule are due to the fact operators proportional to $O_{i}^{2}$ of dimension 4 in the OPE which acquire non-trivial expectation values in the presence of chemical potentials.

\section{Shear sum rule for R-charged M2 and M5-branes}

We will now repeat the analysis and derive the shear sum rules for R-charged M2 and M5-branes. One of our motivations to do this is to verify if the modifications to the sum rules can be explained using the same reasoning employed for the D3-brane case. Indeed we will find that that additional terms in the shear sum rule for the M2-brane can be explained in terms of expectation values of operators in the OPE of the stress tensor. For the M5-brane case we do not find any additional terms in the sum rule and this is consistent with the fact that there are no operators of the requisite dimension in the supergravity which acquires an expectation value. 


\subsection{M2-branes}

We begin with the analysis of the M2-brane at finite chemical potential. In general there are 4 R-charges corresponding to the Cartan's of $S O(8)$ therefore it is possible to turn on 4 independent chemical potentials. The gravity dual of this system is given by the non-extremal R-charged black hole in $A d S_{4}$ [23]. Using the same methods developed for the D3-brane case it is possible to show that the retarded Green's function of the $T_{x y}$ component of the stress tensor satisfies property 1 . To ensure that it satisfies property 2 , we need to subtract the divergent terms at large frequencies. The constant term which arises in the Green's function as $\omega \rightarrow \infty$ essentially is the LHS of the sum rule. Therefore we will directly go ahead and evaluate the divergent pieces as $\omega \rightarrow \infty$ and isolate the term which contributes to the sum rule.

The metric and the gauge field for the R-charged M2-brane with all the four charged turned on is given by

$$
\begin{aligned}
& d s_{4}^{2}=\frac{16\left(\pi T_{0} L\right)^{2}}{9 u^{2}} \mathcal{H}^{1 / 2}\left(-\frac{f}{\mathcal{H}} d t^{2}+d x^{2}+d z^{2}\right)+\frac{L^{2}}{f u^{2}} \mathcal{H}^{1 / 2} d u^{2} \\
& A_{t}^{i}=\frac{4}{3} \pi T_{0} \sqrt{2 k_{i} \prod_{i=1}^{4}\left(1+k_{i}\right)} \frac{u}{H_{i}}, \quad u=\frac{r_{+}}{r}, \quad H_{i}=1+k_{i} u \\
& \mathcal{H}=\prod_{i=1}^{4} H_{i}, \quad f=\mathcal{H}-\prod_{i=1}^{4}\left(1+k_{i}\right) u^{3} .
\end{aligned}
$$

The scalars are given by

$$
X^{i}=\frac{\mathcal{H}^{1 / 4}}{H_{i}(u)} .
$$

The four scalars are not independent and constrained by $X_{1} X_{2} X_{3} X_{4}=1$. The above background is the solution of the equation of motion of the following action

$$
\begin{aligned}
& S=\frac{N^{3 / 2} \sqrt{2}}{24 \pi L^{2}} \int d^{4} x \sqrt{-g} \mathcal{L}, \\
& \mathcal{L}=R-\frac{1}{2}(\partial \vec{\phi})^{2}+V(\phi)-\frac{1}{4} \sum_{i=1}^{4} e^{\vec{a}_{i} \cdot \vec{\phi}}\left(F^{i}\right)^{2},
\end{aligned}
$$

where the fields four fields $X_{i}$ are related to the three independent fields $\vec{\phi}_{i}=$ $\left(\phi_{1}, \phi_{2}, \phi_{3}\right)$ by

$$
\begin{aligned}
& X_{i}=\exp \left(-\frac{1}{2} \vec{a}_{i} \cdot \vec{\phi}\right) \\
& \vec{a}_{1}=(1,1,1), \quad \vec{a}_{2}=(1,-1,-1), \quad \vec{a}_{3}=(-1,1,-1), \vec{a}_{4}=(-1,-1,1) .
\end{aligned}
$$

The scalar potential is given by

$$
V(\phi)=\frac{2}{L^{2}}\left(\cosh \phi_{1}+\cosh \phi_{2}+\cosh \phi_{3}\right) .
$$


Various thermodynamic quantities are summarized as follows: let us first define

$$
T_{0}=\frac{3 r_{+}}{4 \pi L^{2}} .
$$

The energy density and pressure are given by

$$
\begin{aligned}
\epsilon & =\sqrt{2} \pi^{2}\left(\frac{2}{3}\right)^{4} N^{3 / 2} T_{0}^{3} \prod_{i=1}^{4}\left(1+k_{i}\right), \\
P & =\frac{\sqrt{2} \pi^{2}}{3}\left(\frac{2}{3}\right)^{3} N^{3 / 2} T_{0}^{3} \prod_{i=1}^{4}\left(1+k_{i}\right) .
\end{aligned}
$$

The expression for entropy density and temperature is given by

$$
\begin{aligned}
& s=\sqrt{2} \pi^{2}\left(\frac{2}{3}\right)^{3} N^{3 / 2} T_{0}^{2} \prod_{i=1}^{4} \sqrt{1+k_{i}} \\
& T=\frac{T_{0}\left(3+\sum_{j=1}^{4} k_{i}+\sum_{j>i, i, j=1}^{4} k_{i} k_{j}-\prod_{i=1}^{4} k_{i}\right)}{3 \sqrt{\prod_{i=1}^{4}\left(1+k_{i}\right)}} .
\end{aligned}
$$

The charge density and the chemical potential are given by

$$
\begin{aligned}
& \rho_{i}=\sqrt{2} \pi\left(\frac{1}{3}\right)^{3} N^{3 / 2} T_{0}^{2} \sqrt{2 k_{i} \prod_{j=1}^{4}\left(1+k_{j}\right)}, \\
& \mu_{i}=\frac{4 \pi T_{0}}{3} \frac{1}{1+k_{i}} \sqrt{2 k_{i} \prod_{i=1}^{4}\left(1+k_{i}\right) .}
\end{aligned}
$$

To obtain the behaviour of the Green's function as $\omega \rightarrow \infty$, we first write the equation of the minimally coupled scalar in the background given by (5.1) in terms of Fefferman-Graham coordinates. The metric given in (5.1) admits the following form in Fefferman-Graham coordinates.

$$
d s^{2}=\frac{1}{z^{2}}\left(\left(-1+\sum_{i=1}^{\infty} a_{i} z^{i}\right) d t^{2}+\left(1+\sum_{i=1}^{\infty} b_{i} z^{i}\right)\left(d \vec{x}^{2}\right)+d z^{2}\right) .
$$

Here we are working with the scaled variables

$$
z=\frac{r_{+}}{r}, \quad i \lambda=\frac{L^{2}}{r_{+}} \omega .
$$

In the Fefferman-Graham expansion given in (5.10) it can be shown that the terms $a_{1}, b_{1}$ are zero. As we will see subsequently, the asymptotic behaviour of the retarded 
Green's function is essentially determined by the coefficients $a_{2}, b_{2}, a_{3}, b_{3}$ which are evaluated in (A.8). Now the equation for the minimally coupled scalar in this metric (5.10) is given by

$$
\phi^{\prime \prime}-\frac{1}{z}\left(2+\sum_{i=1}^{\infty} \tilde{a}_{i}(z)^{i}\right) \phi^{\prime}-\lambda^{2}\left(1+\sum_{i=1}^{\infty} \tilde{b}_{i}(z)^{i}\right) \phi=0,
$$

where $\tilde{a}_{i}, \tilde{b}_{i}$ can be obtained given $a_{i}, b_{i}$. For the coefficients of interest, this relation is given by

$$
\begin{aligned}
\tilde{a}_{2}=\left(a_{2}-2 b_{2}\right), & \tilde{b}_{2}=a_{2}, \\
\tilde{a}_{3}=\frac{3}{2}\left(a_{3}-2 b_{3}\right), & \tilde{b}_{3}=a_{3} .
\end{aligned}
$$

We now rescale the coordinates by defining

$$
y=\lambda z .
$$

Then the equation for the minimally coupled scalar reduces to

$$
\phi^{\prime \prime}(y)-\frac{1}{y}\left(2+\sum_{i=1}^{\infty} \tilde{a}_{i}\left(\frac{y}{\lambda}\right)^{i}\right) \phi^{\prime}(y)-\left(1+\sum_{i=1}^{\infty} \tilde{b}_{i}\left(\frac{y}{\lambda}\right)^{i}\right) \phi(y)=0 .
$$

Note that at $\lambda$ strictly infinity, the equation reduces to that of the minimally coupled scalar in the $A d S_{4}$ or the $T=0$ background. To solve the equation perturbatively in $\frac{1}{\lambda}$ we define the quantity

$$
g(y)=\frac{\phi^{\prime}}{\phi}
$$

From the expansion of the equation in (5.15), we see that we can expand $g$ as

$$
g=g_{0}+\frac{1}{\lambda^{2}} g_{1}+\frac{1}{\lambda^{3}} g_{2}+\ldots
$$

The equations satisfied by these can be obtain from (5.15) and the first few equations are

$$
\begin{aligned}
& g_{0}^{\prime}+g_{0}^{2}-\frac{2}{y} g_{0}-1=0, \\
& g_{1}^{\prime}+2\left(g_{0}-\frac{1}{y}\right) g_{1}-\tilde{a_{2}} y g_{0}-\tilde{b_{2}} y^{2}=0, \\
& g_{2}^{\prime}+2\left(g_{0}-\frac{1}{y}\right) g_{2}-\tilde{a_{3}} y^{2} g_{0}-\tilde{b_{3}} y^{3}=0 .
\end{aligned}
$$

The two independent solutions for the first equation in (5.18) are

$$
g_{0}^{(1)}=-\frac{K_{1 / 2}(y)}{K_{3 / 2}(y)}=-\frac{y}{1+y}, \quad g_{0}^{(2)}=\frac{I_{1 / 2}(y)}{I_{3 / 2}(y)}=\frac{x \sinh x}{x \cosh x-\sinh x} .
$$


As in the case of the D3-branes we can solve for the two independent solutions order by order in $\frac{1}{\lambda}$ around these solutions. Just as in the case of the D3-brane situation we find that the solution around $g_{0}^{(1)}$ determines the leading asymptotic behaviour of the Green's function as $\lambda \rightarrow \infty$. This is because of the condition that at $\lambda \rightarrow \infty$, the solution must reduce to that of the $T=0$ case. $I_{3 / 2}(y)$ is a growing solution as $y \rightarrow \infty$ and thus is not allowed when $T=0$. The leading corrections around the solution $g_{0}^{(0)}$ is given by

$$
\begin{aligned}
& g_{1}=-y^{2}\left(\frac{a_{2}}{2}+b_{2} \frac{2 y+3}{2(1+y)^{2}}\right), \\
& g_{2}=\frac{3}{4}\left(a_{3}-2 b_{3}\right) y^{2}-\frac{a_{3} y^{2}}{8(1+y)^{2}}\left(9+18 y+14 y^{2}+4 y^{3}\right) .
\end{aligned}
$$

The retarded Green's function is then given by

$$
\begin{aligned}
\lim _{\omega \rightarrow \infty} G_{R}(\omega, T)= & \lim _{\omega \rightarrow \infty} \frac{\sqrt{2} N^{3 / 2} r_{+}^{3}}{24 \pi L^{6}} \lim _{y \rightarrow 0} \frac{1}{y^{2}}\left(\lambda^{3} g_{0}^{(1)}(y)+\lambda g_{1}^{(1)}(y)+g_{2}^{(1)}(y)\right) \\
& +G_{\text {contact }}+G_{\text {counter }}
\end{aligned}
$$

Substituting the form for $g_{1}^{(1)}$ and $g_{2}^{(1)}$ in the limit we have

$$
\lambda \lim _{y \rightarrow 0} \frac{g_{1}^{(1)}(y)}{y^{2}}=-\frac{\lambda}{2}\left(a_{2}+3 b_{2}\right), \quad \lim _{y \rightarrow 0} \frac{g_{2}^{(1)}(y)}{y^{2}}=-\frac{3}{8}\left(a_{3}+4 b_{3}\right) .
$$

From the expressions in (5.22), it is clear that there are two sources of divergence. The term proportional to $\lambda^{3}$ which is identical to the contribution of the $T=0$ Green's function. The second divergence is due to the term proportional to $\lambda$. This gives a linear divergence in $\omega$ and we need to subtract this also along with the constant term from the limit of $g_{2}^{(1)}$ to ensure that property 2 . is satisfied. Therefore we define

$$
\begin{aligned}
\delta G_{R}(\omega, T)= & G_{R}(\omega, T)-G_{R}(\omega, 0)-G_{\text {contact }} \\
& +i \delta \rho(\omega)+\frac{\sqrt{2} N^{3 / 2} r_{+}^{3}}{24 \pi L^{6}} \frac{3}{8}\left(a_{3}+4 b_{3}\right) .
\end{aligned}
$$

where

$$
\begin{aligned}
& \delta \rho(\omega)=-\frac{\sqrt{2} N^{3 / 2} r_{+}^{2} \omega}{24 \pi L^{4}} \frac{1}{2}\left(a_{2}+3 b_{2}\right), \\
= & \frac{\sqrt{2} \pi T_{0}^{2} N^{3 / 2} \omega}{432}\left(\left(k_{1}+k_{2}-k_{3}-k_{4}\right)^{2}+\left(k_{1}-k_{2}+k_{3}-k_{4}\right)^{2}+\left(k_{1}-k_{2}-k_{3}+k_{4}\right)^{2}\right) .
\end{aligned}
$$

Note that we have subtracted the required terms to ensure that $\delta G_{R}(\omega, T)$ obeys property 2 to apply Cauchy's theorem. The RHS of the sum rule therefore involves the following integrand

$$
\begin{aligned}
\operatorname{Im} \delta G_{R}(\omega, T) & =\rho(\omega)+\delta \rho(\omega)-\rho(\omega, 0), \\
& =\tilde{\rho}(\omega, T)-\rho(\omega, 0) .
\end{aligned}
$$


The LHS of the sum rule is obtained by evaluating

$$
\begin{aligned}
\delta G_{R}(0, T) & =\frac{\sqrt{2} N^{3 / 2} r_{+}^{3}}{24 \pi L^{6}} \frac{3}{8}\left(a_{3}+b_{3}\right), \\
& =\frac{3}{8} \epsilon+\frac{\sqrt{2} \pi N^{3 / 2} T_{0}^{3}}{216}\left(k_{1}+k_{2}-k_{3}-k_{4}\right)\left(k_{1}-k_{2}+k_{3}-k_{4}\right)\left(k_{1}-k_{2}-k_{3}+k_{4}\right) .
\end{aligned}
$$

Here we have substituted the values of $a_{3}, a_{4}$ from (A.8). Thus the sum rule for the M2-brane theory in the presence of chemical potential is given by

$$
\begin{aligned}
& \frac{3}{8} \epsilon+\frac{\sqrt{2} \pi^{2} N^{3 / 2} T_{0}^{3}}{216}\left(k_{1}+k_{2}-k_{3}-k_{4}\right)\left(k_{1}-k_{2}+k_{3}-k_{4}\right)\left(k_{1}-k_{2}-k_{3}+k_{4}\right) \\
& =\frac{1}{\pi} \int_{-\infty}^{\infty} \frac{d \omega}{\omega}\left(\tilde{\rho}(\omega)-\rho_{T=0}(\omega)\right) .
\end{aligned}
$$

Note that $\tilde{\rho}(\omega)$ is defined by

$$
\tilde{\rho}(\omega)_{T}=\rho(\omega)_{T}+\delta \rho(\omega),
$$

where $\delta \rho(\omega)$ is given in (5.24).

From the above analysis we see that the sum rule is essentially due to the high frequency behaviour of the Green's function. High frequency behaviour of the Green's function can be extracted from the OPE of the stress tensor. We will now show that the linear divergence in $\omega$ and the additional term involving the chemical potentials in the LHS of the sum rule are due to the presence of certain operators in the OPE which get expectation values in the presence of the sum rule. The general form for the OPE of the stress tensor in this case is given by

$$
T_{\mu \nu}(x) T_{\rho \sigma}(0) \sim C_{T} \frac{I_{\mu \nu, \rho \sigma}}{x^{6}}+\hat{A}_{\mu \nu \rho \sigma \alpha \beta}(x) T_{\alpha \beta}(0)+B_{\mu \nu \rho \sigma}^{a}(x) \mathcal{O}_{a}(0) .
$$

Here we have included additional terms in the OPE which arise if there are scalars denoted by $\mathcal{O}_{a}$ are present in the theory and the three point function $\left\langle T_{\mu \nu} T_{\rho \sigma} \mathcal{O}_{a}\right\rangle$ is non-zero. Note that now the stress tensor is a dimension 3 operator in this case. From a similar analysis as in the case of the D3-brane case, we see that a linear divergence can be explained due the presence of operators $\mathcal{O}$ of dimension 1 in the OPE. The constant terms must arise from operators of dimension 3. Since the expectation value of the stress tensor accounts for the term $\frac{3}{8} \epsilon$ in the sum rule, the additional terms in the LHS of the sum rule must arise from other operators $\mathcal{O}_{a}$ of dimension 3. Note that the $\omega$ divergence in (5.24) and the additional term present in the LHS of the sum rule in (5.27) vanish when all the chemical potentials are equal. In such a situation from the solution given in (5.1) one sees that there is non-trivial gauge field but the scalars given in (5.2) become trivial. Thus the presence of expectation values of these operators must be due to the scalars. 
Let us now examine the scalars in detail. Expanding the scalar potential given in (5.5) to quadratic order we find that all the three scalars $\phi_{i}$ are of equal mass with the mass given by

$$
m^{2} L^{2}=-2
$$

The scaling dimension of the operator dual to the scalars can be found by the massdimension relation

$$
\Delta(\Delta-3)=m^{2} L^{2} .
$$

From this equation we see that the dimension of the operators $\Delta$ can be chosen to be 1 or 2 . When $\Delta$ is chosen to be 2 the quantization of the scalar is conventionally known as the standard quantization. One the other hand when $\Delta$ is chosen to be 1 the quantization of the scalar obey the alternate quantization. For the neutral scalars in the M2-brane background it has been seen earlier [24, 25 that one must chose the alternate quantization. Let us denote the three operators dual to these bulk scalar fields as $O_{i}$ which have dimension 1 each. We now read out the expectation values of these operators in the non-extremal M2-brane background following [20]. The expansion of $\phi_{1}$ close to the boundary is of the form

$$
\phi_{i}=\alpha_{i} \frac{1}{r}+L^{2} \beta_{i} \frac{1}{r^{2}}+\cdots .
$$

The factor of $L$ has been re-instated so that $\beta$ has the appropriate dimensions. For the alternate quantization, the expectation value of the operators is given by the coefficient $\frac{N^{3 / 2} \sqrt{2}}{24 \pi L^{2}} \alpha_{i}$. Expanding the scalar fields $\phi_{i}$ defined in (5.4) near the boundary and using (5.2) we obtain

$$
\begin{aligned}
& \left\langle O_{1}\right\rangle=-\left(\frac{N^{\frac{3}{2}} \sqrt{2}}{24 \pi}\right) \frac{r_{+}}{2 L^{2}}\left(k_{1}+k_{2}-k_{3}-k_{4}\right), \\
& \left\langle O_{2}\right\rangle=-\left(\frac{N^{\frac{3}{2}} \sqrt{2}}{24 \pi}\right) \frac{r_{+}}{2 L^{2}}\left(k_{1}-k_{2}+k_{3}-k_{4}\right), \\
& \left\langle O_{3}\right\rangle=-\left(\frac{N^{\frac{3}{2}} \sqrt{2}}{24 \pi}\right) \frac{r_{+}}{2 L^{2}}\left(k_{1}-k_{2}-k_{3}+k_{4}\right) .
\end{aligned}
$$

From these expectation values we see that the candidate operators which are responsible for the linear $\omega$ divergence given in (5.24) are proportional $O_{i}^{2}$. Indeed taking the sum or squares of these operators we obtain

$$
\begin{aligned}
& \sum_{i=1}^{2}\left\langle O_{i}^{2}\right\rangle=\sum_{i=1}^{3}\left\langle O_{i}\right\rangle^{2} \\
& =\frac{N^{3}}{648}\left(\left(k_{1}+k_{2}-k_{3}-k_{4}\right)^{2}+\left(k_{1}-k_{2}+k_{3}-k_{4}\right)^{2}+\left(k_{1}-k_{2}-k_{3}+k_{4}\right)^{2}\right) .
\end{aligned}
$$


In the first line we have assumed large $N$ factorization. Note that the last line in (5.34) is proportional to the linear omega divergence seen in (5.24). The factors of $N$ will present in (5.34) will agree with that present in (5.24) if one assumes the structure constant $B_{\mu \nu \rho}^{a}$ with the normalization of the operators used scales as $N^{-3 / 2}$. Indeed this is what is seen from the gravity analysis ${ }^{6}$. We can also obtain the additional term present in the LHS of the sum rule given in (5.27). Note that for this we require an operator of dimension 3 . We see that the required operator is proportional to $\mathrm{O}_{1} \mathrm{O}_{2} \mathrm{O}_{3}$. Indeed we find

$$
\begin{aligned}
& \left\langle O_{1} O_{2} O_{2}\right\rangle=\left\langle O_{1}\right\rangle\left\langle O_{2}\right\rangle\left\langle O_{2}\right\rangle, \\
& =\frac{N^{\frac{9}{2}} \sqrt{2}}{2^{5} 3^{6}}\left(k_{1}+k_{2}-k_{3}-k_{4}\right)\left(k_{1}-k_{2}+k_{3}-k_{4}\right)\left(k_{1}-k_{2}-k_{3}+k_{4}\right) .
\end{aligned}
$$

which proportional to the additional term in (5.27) with the exception of the $N$ scaling. The $N$ scaling can be understood if one assumes that the structure constant $B_{\mu \nu \rho \sigma}^{a}$ scales as $N^{-3}$ for these operators. Thus the additional term in the LHS of the sum rule (5.27) is due to these additional operators in the OPE which gain expectation value in presence of the chemical potential.

\subsection{M5-branes}

In this section we examine the dual of M5-brane at finite chemical potential and finite temperature and re-derive the shear sum rule. Since there are 2 R-charges corresponding to the Cartan's of $\mathrm{SO}(5)$ it is possible to turn on 2 independent chemical potential,since there are no constrain equation relating this two R-charges. Using the differential equation of the massless minimally coupled scalar in this background we obtain the retarded Green's function of the $T_{x y}$ component of the stress tensor. Examining this differential equation and the same method employed for he D3-brane case it can be shown that the regulated Green's function satisfies both property 1 and property 2 which are necessary for deriving the sum rule. However as will see the shear sum rule for the case of M5-brane is not modified. We argue that this must be the case since there are no scalars in the M5-brane gravity background which have the appropriate dimensions to modify the OPE of the stress tensor.

The metric and the gauge field for the R-charged M5-brane with all the charges turned on is given by 23]

$$
\begin{aligned}
& d s_{7}^{2}=\frac{4\left(\pi T_{0} L\right)^{2}}{9 u} \mathcal{H}^{1 / 5}\left(-\frac{f}{\mathcal{H}} d t^{2}+d x_{1}^{2}+\cdots+d x_{4}^{2}+d z^{2}\right)+\frac{L^{2}}{4 f u^{2}} \mathcal{H}^{1 / 5} d u^{2}, \\
& A_{t}=\frac{2}{3} \pi T_{0} \sqrt{2 k_{i} \prod_{i=1}^{2}\left(1+k_{i}\right)} \frac{u^{2}}{H_{i}}, \quad H_{i}=1+k_{i} u^{2},
\end{aligned}
$$

\footnotetext{
${ }^{6}$ Since we do not have an explicit theory for the M2-branes at present we cannot understand the $N$ scalings from a field theory analysis for the M2-branes.
} 


$$
\begin{aligned}
& T_{0}=\frac{3 r_{+}}{2 \pi L^{2}}, \quad \epsilon=\frac{5 \pi^{3}}{2} \frac{2^{7}}{3^{7}} N^{3} T_{0}^{6} \prod_{i=1}^{2}\left(1+k_{i}\right) \\
& H_{i}=1+k_{i} u^{2}, \quad \mathcal{H}=\prod_{i=1}^{2} H_{i}, \quad f=\mathcal{H}-\prod_{i=1}^{2}\left(1+k_{i}\right) u^{3} .
\end{aligned}
$$

The background solution for the two scalars are given by

$$
X^{i}=\frac{\mathcal{H}^{2 / 5}}{H_{i}(u)}
$$

where $u=\frac{r_{+}^{2}}{r^{2}}$. The above background is the solution of the equation of motion of the action is

$$
\begin{aligned}
& S=\frac{N^{3}}{6 \pi^{3} L^{5}} \int d^{7} x \sqrt{-g} \mathcal{L}, \\
& \mathcal{L}=R-\frac{1}{2}(\partial \vec{\phi})^{2}+V(\phi)-\frac{1}{4} \sum_{i=1}^{2} e^{\vec{a}_{i} \cdot \vec{\phi}}\left(F^{i}\right)^{2} .
\end{aligned}
$$

Where the two scalar fields $X_{i}^{\prime} s$ are related to $\vec{\phi}=\left(\phi_{1}, \phi_{2}\right)$ b;y

$$
\begin{aligned}
X_{i} & =e^{-\frac{1}{2} \vec{a} \cdot \vec{\phi}}, \\
\overrightarrow{a_{1}} & =\left(\sqrt{2}, \sqrt{\frac{2}{5}}\right), \quad \overrightarrow{a_{2}}=\left(-\sqrt{2}, \sqrt{\frac{2}{5}}\right) .
\end{aligned}
$$

Note that the two scalars $X_{i}$ are independent here, unlike the situation in the case of the D3-branes and M2-branes. The scalar potential $V$ is given by

$$
V=\frac{4}{L^{2}}\left(-4 X_{1} X_{2}-2 X_{1}^{-1} X_{2}^{-2}-2 X_{1}^{-2} X_{2}^{-1}+\frac{1}{2}\left(X_{1} X_{2}\right)^{-4}\right) .
$$

The leading terms in the large frequency expansion of the equation for $x y$ component of the metric field in the Fefferman-Graham coordinate system is given by

$$
\phi^{\prime \prime}-\frac{1}{y}\left(5+\frac{3\left(a_{3}-5 b_{3}\right) y^{6}}{\lambda^{6}}\right) \phi^{\prime}-\left(1+a_{3} \frac{y^{6}}{\lambda^{6}}\right) \phi=0 .
$$

where we have defined

$$
y=\lambda z=\lambda \frac{r_{+}}{r}, \quad i \lambda=\frac{L^{2}}{r_{+}} \omega .
$$

The values of $a_{3}, b_{3}$ are evaluated in in (A.11). Let us define

$$
\tilde{a}_{3}=3\left(a_{3}-5 b_{3}\right), \quad \tilde{b}_{3}=a_{3} .
$$


Since the Green's function is proportional to $g=\frac{\phi^{\prime}}{\phi}$, we can write the equation in (5.41) as

$$
g^{\prime}+g^{2}-\frac{1}{y}\left(5+\frac{\tilde{a}_{3} y^{6}}{\lambda^{6}}\right) g-\left(1+\tilde{b}_{3} \frac{y^{6}}{\lambda^{6}}\right)=0 .
$$

We then find the solution to the leading order by writing $g$ as

$$
g=g_{0}+\frac{1}{\lambda^{6}} g_{1}+\cdots
$$

The equations satisfied by each of the terms in the expansion of $g$ can be obtain from (5.44). They are given by

$$
\begin{aligned}
& g_{0}^{\prime}+g_{0}^{2}-\frac{5}{y} g_{0}-1=0, \\
& g_{1}^{\prime}+2 g_{0} g_{1}-\frac{5}{y} g_{1}-\tilde{a}_{3} y^{5} g_{0}-\tilde{b}_{3} y^{6}=0 .
\end{aligned}
$$

The relevant solution for our analysis are

$$
g_{0}^{(1)}=-\frac{K_{2}(y)}{K_{3}(y)}, \quad g_{1}^{(1)}=\frac{\tilde{a}_{3} y^{5}}{2}+\frac{\tilde{b}_{3} y^{7}}{14}\left(1-\frac{K_{4}^{2}}{K_{3}^{2}}\right) .
$$

The retarded Green's function is then given by

$$
\begin{aligned}
\lim _{\omega \rightarrow \infty} G_{R}(\omega, T)= & \lim _{\omega \rightarrow \infty} \frac{N^{3} r_{+}^{6}}{6 \pi^{3} L^{12}} \lim _{y \rightarrow 0} \frac{1}{y^{2}}\left(\lambda^{6} g_{0}^{(1)}(y)+g_{1}^{(1)}(y)\right) \\
& +G_{\text {contact }}+G_{\text {counter }}
\end{aligned}
$$

The term proportional to $\lambda^{6}$ which is identical to the contribution of the $T=0$ retarded Green's function. It is divergent in the limit $\lambda \rightarrow \infty$. To ensure that the property 2 is satisfied we need to subtract the divergent piece and the constant pieces from the Green's function to write the regulated Green's function. Therefore we define

$$
\begin{aligned}
\delta G_{R}(\omega, T)= & G_{R}(\omega, T)-G_{R}(\omega, 0)-G_{\text {contact }} \\
& +\frac{N^{3}}{3 \pi^{3}} \frac{15 r_{+}^{6}}{14 L^{12}}\left(7 b_{3}+a_{3}\right) .
\end{aligned}
$$

The LHS of the sum rule is obtain by evaluating

$$
\begin{aligned}
\delta G_{R}(0, T) & =\frac{N^{3}}{3 \pi^{3}} \frac{15}{7}\left(\frac{2 \pi T_{0}}{3}\right)^{6}\left(1+\kappa_{1}\right)\left(1+\kappa_{2}\right), \\
& =\frac{3}{7} \epsilon .
\end{aligned}
$$

Here we have substituted the values of $a_{3}, b_{3}$ from (A.11). Thus the sum rule for the M5-brane in the presence of chemical potential is given by

$$
\frac{3}{7} \epsilon=\frac{1}{\pi} \int_{-\infty}^{\infty} \frac{d \omega}{\omega}\left(\rho(\omega)-\rho_{T=0}(\omega)\right) .
$$


Note that for the case of R-charged M5-branes there is no additional contribution in the shear sum rule compared to uncharged case. This fact can be understood as follows. Let us examine the masses of the scalars in the theory. From expanding the potential for the scalars in (5.40) we see that the two scalars $\phi_{i}$ in the theory have mass given by by

$$
m^{2} L^{2}=-8
$$

From the mass-dimension relation we see that there are two possible choices of mass for the operators dual to these. They are $\Delta=2$ or $\Delta=4$. The stress tensor for the 6-dimensional theory of the M5-brane is an operator of dimension 6. Thus if there are terms which are finite in the large frequency expansion of the OPE, they must be of dimension 6 . The general form for the asymptotic expansion of the scalar field in this case is given by

$$
\phi_{i}=\frac{\alpha_{i}}{r^{2}}+\frac{\beta_{i}}{r^{4}}+\cdots
$$

From examining the background value for the scalar field given in (5.37) we see that for both the scalar fields, $\alpha_{i}=0$ and $\beta_{i} \neq 0$. In the standard quantization of the scalar fields, the dual operators have dimension $\Delta=4$ and the expectation value of the scalar field is proportional to the value of $\beta_{i}$. But in this situation there is no operator which has a dimension $\Delta=6$ which gains expectation value. This implies there is no finite term in the large frequency expansion of the OPE of the stress tensor ${ }^{7}$. If we choose the alternate quantization, then the operators dual to the fields $\phi_{i}$ have dimension 2, but from the expansion of the scalar field we see that their expectation value is proportional to the value of $\alpha_{i}$ which is zero. Thus in the alternate quantization the operator dual to the scalar field does not gain expectation value. Therefore it cannot contribute to either a divergence or the finite term in the OPE of the stress tensor. These arguments imply that there is there is no correction to the sum rule which is consistent with our explicit calculation.

\section{Conclusions}

By examining the shear sum rule for the case of R-charged D3-branes and M2branes we arrive at the general observation that sum rule acquires additional terms proportional to the chemical potential. These additional terms are the result of expectation values of operators of dimension 4 and dimension 3 for the case of D3branes and M2-branes respectively. For the case of the M5-brane this no change in the sum rule at finite chemical potential. This is explained by the fact that there is no operator of dimension 6 whose expectation value is turned on at finite chemical

\footnotetext{
${ }^{7}$ The operator dual to the scalar field with dimension $\Delta=4$ can give rise to a $\omega^{2}$ divergence if there the 3 point function of this operator with the stress tensor $\left\langle T_{\mu \nu} T_{\rho \sigma} \mathcal{O}\right\rangle$ is not zero. From our gravity analysis since there is no divergence it is clear that this three point function vanishes.
} 
potential. Our analysis indicates that the LHS of the sum rule contains information of the OPE coefficients of the stress tensor with scalar operators of the appropriate dimensions. Thus this approach can be used to compute three point of functions of the stress tensor with these operators from gravity. This will involve writing down the relating the coefficient $B_{\mu \nu \rho \sigma}^{a}$ given in (4.60) in terms of the coefficient which occurs in the three point function using a similar analysis of [16]. It will be interesting to explicitly check the three point function obtained in this manner against a direct evaluation three point functions from gravity.

Another direction to extend this work would be to examine sum rules for the R-charge correlators in $\mathcal{N}=4$ Yang-Mills at finite chemical potential. It will be interesting to see if the effects of triangle anomalies can be seen from the sum rule just as hydrodynamics was modified on the account of triangle anomalies [26]. Finally we mention that chemical potential is an important parameter in the QCD phase diagram and it will be interesting to see if sum rules can be derived in this context.

\section{Acknowledgments}

We wish to thank Avinash Dhar, Rajesh Gopakumar, Kavita Jain, Gautam Mandal, Shiraz Minwalla, Ashoke Sen and Dam Son for useful discussions. S. J would like to thank CHEP, IISc, Bangalore for hospitality where part of this work was done. The work of J.R.D is partially supported by the Ramanujan fellowship DST-SR/S2/RJN$59 / 2009$.

\section{A. Fefferman-Graham coordinates}

In this appendix we will detail the co-ordinate transformation which takes the metrics discussed in this paper to the Fefferman-Graham coordinates. We will see that for the non-extremal R-charged D3 and M5-branes, the metric in Fefferman-Graham co-ordinates takes the form

$$
d s^{2}=\frac{1}{z^{\prime 2} \tilde{L}^{2}}\left(\left(-1+\sum_{i=1}^{\infty} a_{i}\left(z^{\prime 2}\right)^{i}\right) d t^{2}+\left(1+\sum_{i=1}^{\infty} b_{i}\left(z^{\prime 2}\right)^{i}\right)\left(d \vec{x}^{2}\right)+d z^{\prime 2}\right) .
$$

For the case of the non-extremal R-charged M2-brane the metric in FeffermanGraham co-ordinates takes the form

$$
d s^{2}=\frac{1}{z^{\prime 2} \tilde{L}^{2}}\left(\left(-1+\sum_{i=1}^{\infty} a_{i}\left(z^{\prime}\right)^{i}\right) d t^{2}+\left(1+\sum_{i=1}^{\infty} b_{i}\left(z^{\prime}\right)^{i}\right)\left(d \vec{x}^{2}\right)+d z^{\prime 2}\right)
$$

here $\tilde{L}=L / r_{+}$. In this appendix we will evaluate the coefficients $a_{i}, b_{i}$ which are necessary to obtain the sum rule. 


\section{Non-extremal R-charged D3-brane}

The metric R-charged D3-brane metric given in in (4.1) can be transformed to the

Fefferman-Graham form by first redefining the co-ordinate $z=\frac{r_{+}}{r}$ in terms of the Fefferman-Graham co-ordinate $z^{\prime}$ as a power series

$$
\begin{aligned}
z & =\Omega\left(z^{\prime}\right), \\
& =z^{\prime}\left(1+\alpha z^{\prime 2}+\beta z^{\prime 4}+O\left(z^{\prime 6}\right)\right) .
\end{aligned}
$$

After expanding the coefficients of the metric (4.1) as a power series in $z$ around $z=0$ we substitute the expansion (A.3) in the metric (4.1). We then determine the coefficients $\alpha, \beta, \cdots$ by requiring that the coefficient of the $d z^{\prime 2}$ term reduces to $\frac{1}{z^{\prime 2}}$. Performing this procedure to $O\left(z^{2}\right)$ results in the following values of $\alpha, \beta$

$$
\begin{aligned}
& \alpha=\frac{1}{6}\left(k_{1}+k_{2}+k_{3}\right), \\
& \beta=\frac{1}{72}\left\{\left(k_{1}^{2}+k_{2}^{2}+k_{3}^{2}\right)-\left(k_{1} k_{2}+k_{2} k_{3}+k_{3} k_{1}\right)-9\left(1+k_{1}+k_{2}+k_{3}+k_{1} k_{2} k_{3}\right)\right\} .
\end{aligned}
$$

Finally we substitute these values back in the remaining coefficients of the metric to obtain

$$
\begin{aligned}
a_{1} & =0, \quad b_{1}=0, \\
a_{2} & =\frac{1}{36}\left\{27\left(1+k_{1}+k_{2}+k_{3}+k_{1} k_{2} k_{3}\right)+25\left(k_{1} k_{2}+k_{2} k_{3}+k_{3} k_{1}\right)\right. \\
& \left.+2\left(k_{1}^{2}+k_{2}^{2}+k_{3}^{2}\right)\right\}, \\
b_{2} & =\frac{1}{36}\left\{9\left(1+k_{1}+k_{2}+k_{3}+k_{1} k_{2} k_{3}\right)+11\left(k_{1} k_{2}+k_{2} k_{3}+k_{3} k_{1}\right)\right. \\
& \left.-2\left(k_{1}^{2}+k_{2}^{2}+k_{3}^{2}\right)\right\} .
\end{aligned}
$$

\section{Non-extremal R-charged M2-brane}

We now perform the same procedure for the metric given in (5.1). Let us define the co-ordinate $z$ as

$$
\begin{aligned}
z & =\Omega\left(\overline{z^{\prime}}\right), \\
& =z^{\prime}\left(1+\alpha z^{\prime}+\beta z^{\prime 2}+\gamma z^{\prime 3}+O\left(z^{\prime 4}\right)\right) .
\end{aligned}
$$

Demanding that the coefficient of $d z^{\prime 2}$ to be of the form of $\frac{1}{z^{\prime 2}}$ to $O\left(z^{\prime 4}\right)$ in the new coordinate system determines $\alpha, \beta, \gamma$. They are given by

$$
\begin{aligned}
\alpha & =\frac{1}{4}\left(k_{1}+k_{2}+k_{3}+k_{4}\right), \\
\beta & =\frac{1}{64}\left\{\left(k_{1}^{2}+k_{2}^{2}+k_{3}^{2}+k_{4}^{2}\right)+10\left(k_{1} k_{2}+k_{1} k_{3}+k_{1} k_{4}+k_{2} k_{3}+k_{2} k_{4}+k_{3} k_{4}\right)\right\}, \\
\gamma & =\frac{1}{384}\left\{\left(k_{1}^{3}+k_{2}^{3}+k_{3}^{3}+k_{4}^{3}\right)\right. \\
& +11\left(k_{1}^{2} k_{2}+k_{2}^{2} k_{1}+k_{1}^{2} k_{3}+k_{3}^{2} k_{1}+k_{1}^{2} k_{4}+k_{4}^{2} k_{1}+k_{2}^{2} k_{3}+k_{3}^{2} k_{2}+k_{2}^{2} k_{4}+k_{4}^{2} k_{2}+k_{3}^{2} k_{4}+k_{4}^{2} k_{3}\right) \\
& -2\left(k_{1} k_{2} k_{3}+k_{1} k_{2} k_{4}+k_{1} k_{3} k_{4}+k_{2} k_{3} k_{4}\right)-64\left(1+k_{1}+k_{2}+k_{3}+k_{4}\right. \\
& \left.\left.+k_{1} k_{2}+k_{1} k_{3}+k_{1} k_{4}+k_{2} k_{3}+k_{2} k_{4}+k_{3} k_{4}+k_{1} k_{2} k_{3} k_{4}\right)\right\} .
\end{aligned}
$$


We then substitute these values back in the other coefficients of the metric to determine the values of $a_{i}, b_{i}$ which results in the following values.

$$
\begin{aligned}
a_{1} & =b_{1}=0, \\
a_{2} & =\frac{1}{32}\left\{3\left(k_{1}^{2}+k_{2}^{2}+k_{3}^{2}+k_{4}^{2}\right)-2\left(k_{1} k_{2}+k_{1} k_{3}+k_{1} k_{4}+k_{2} k_{3}+k_{2} k_{4}+k_{3} k_{4}\right)\right\}, \\
a_{3} & =\frac{1}{24}\left\{-\left(k_{1}^{3}+k_{2}^{3}+k_{3}^{3}+k_{4}^{3}\right)+14\left(k_{1} k_{2} k_{3}+k_{1} k_{2} k_{4}+k_{1} k_{3} k_{4}+k_{2} k_{3} k_{4}\right)\right. \\
& +\left(k_{1}^{2} k_{2}+k_{2}^{2} k_{1}+k_{1}^{2} k_{3}+k_{3}^{2} k_{1}+k_{1}^{2} k_{4}+k_{4}^{2} k_{1}+k_{2}^{2} k_{3}+k_{3}^{2} k_{2}+k_{2}^{2} k_{4}+k_{4}^{2} k_{2}+k_{3}^{2} k_{4}+k_{4}^{2} k_{3}\right) \\
& \left.+16\left(1+k_{1}+k_{2}+k_{3}+k_{4}+k_{1} k_{2}+k_{1} k_{3}+k_{1} k_{4}+k_{2} k_{3}+k_{2} k_{4}+k_{3} k_{4}+k_{1} k_{2} k_{3} k_{4}\right)\right\}, \\
b_{2} & =-\frac{1}{32}\left\{3\left(k_{1}^{2}+k_{2}^{2}+k_{3}^{2}+k_{4}^{2}\right)-2\left(k_{1} k_{2}+k_{1} k_{3}+k_{1} k_{4}+k_{2} k_{3}+k_{2} k_{4}+k_{3} k_{4}\right)\right\}, \\
b_{3} & =\frac{1}{24}\left\{\left(k_{1}^{3}+k_{2}^{3}+k_{3}^{3}+k_{4}^{3}\right)+10\left(k_{1} k_{2} k_{3}+k_{1} k_{2} k_{4}+k_{1} k_{3} k_{4}+k_{2} k_{3} k_{4}\right)\right. \\
& -\left(k_{1}^{2} k_{2}+k_{2}^{2} k_{1}+k_{1}^{2} k_{3}+k_{3}^{2} k_{1}+k_{1}^{2} k_{4}+k_{4}^{2} k_{1}+k_{2}^{2} k_{3}+k_{3}^{2} k_{2}+k_{2}^{2} k_{4}+k_{4}^{2} k_{2}+k_{3}^{2} k_{4}+k_{4}^{2} k_{3}\right) \\
& \left.+8\left(1+k_{1}+k_{2}+k_{3}+k_{4}+k_{1} k_{2}+k_{1} k_{3}+k_{1} k_{4}+k_{2} k_{3}+k_{2} k_{4}+k_{3} k_{4}+k_{1} k_{2} k_{3} k_{4}\right)\right\} .
\end{aligned}
$$

\section{Non-extremal R-charged M5-brane}

We follow the same procedure again. We first expand the metric around $z=0$, retaining terms to order $\mathcal{O}\left(z^{6}\right)$ and then apply the coordinate transformation

$$
\begin{aligned}
z & =\Omega\left(z^{\prime}\right), \\
& =z^{\prime}\left(1+\alpha z^{\prime 2}+\beta z^{4}+\gamma \bar{z}^{6}+O\left(z^{\prime 7}\right)\right) .
\end{aligned}
$$

By demanding that the coefficient of $d z^{\prime 2}$ is of the form of $\frac{1}{z^{\prime 2}}$ to $O\left(z^{17}\right)$ in the new coordinate system we can determine $\alpha, \beta$ and $\gamma$. They are given by

$$
\alpha=0, \quad \beta=\frac{k_{1}+k_{2}}{10}, \quad \gamma=-\frac{\left(1+k_{1}\right)\left(1+k_{2}\right)}{12} .
$$

Substituting these values back in the metric we determine the other coefficients of the metric to be given by

$$
\begin{aligned}
a_{1}=b_{1}=0, & a_{2}=b_{2}=0, \\
a_{3}=\frac{5}{6}\left(1+k_{1}\right)\left(1+k_{2}\right), & b_{3}=\frac{1}{6}\left(1+k_{1}\right)\left(1+k_{2}\right) .
\end{aligned}
$$

\section{References}

[1] R. Baier, R-charge thermodynamical spectral sum rule in $N=4$ Yang-Mills theory, arXiv:0910.3862.

[2] D. Kharzeev and K. Tuchin, Bulk viscosity of QCD matter near the critical temperature, JHEP 0809 (2008) 093, arXiv:0705.4280. 
[3] F. Karsch, D. Kharzeev, and K. Tuchin, Universal properties of bulk viscosity near the QCD phase transition, Phys.Lett. B663 (2008) 217-221, arXiv:0711.0914.

[4] H. B. Meyer, The Bulk Channel in Thermal Gauge Theories, JHEP 1004 (2010) 099, arXiv:1002.3343.

[5] H. B. Meyer, Lattice Gauge Theory Sum Rule for the Shear Channel, Phys.Rev. D82 (2010) 054504, arXiv:1005.2686.

[6] R. A. Ferrell and R. E. Glover, Conductivity of Superconducting Films: A Sum Rule, Phys.Rev. 109 (1958) 1398-1399.

[7] M. Tinkham and R. A. Ferrell, Determination of the superconducting skin depth from the energy gap and sum rule, Phys. Rev. Lett. 2 (Apr, 1959) 331-333.

[8] P. Romatschke and D. T. Son, Spectral sum rules for the quark-gluon plasma, Phys.Rev. D80 (2009) 065021, arXiv:0903.3946].

[9] T. Springer, C. Gale, S. Jeon, and S. H. Lee, A shear spectral sum rule in a non-conformal gravity dual, Phys.Rev. D82 (2010) 106005, arXiv:1006.4667.

[10] T. Springer, C. Gale, and S. Jeon, Bulk spectral functions in single and multi-scalar gravity duals, Phys.Rev. D82 (2010) 126011, arXiv:1010.2760.

[11] D. R. Gulotta, C. P. Herzog, and M. Kaminski, Sum Rules from an Extra Dimension, JHEP 1101 (2011) 148, arXiv:1010.4806.

[12] G. Policastro, D. T. Son, and A. O. Starinets, From AdS / CFT correspondence to hydrodynamics, JHEP 0209 (2002) 043, hep-th/0205052.

[13] P. K. Kovtun and A. O. Starinets, Quasinormal modes and holography, Phys.Rev. D72 (2005) 086009, hep-th/0506184.

[14] D. T. Son and A. O. Starinets, Minkowski space correlators in AdS / CFT correspondence: Recipe and applications, JHEP 0209 (2002) 042, hep-th/0205051.

[15] V. I. Arnol'd, Ordinary differential equations, Springer-Verlag, New York.

[16] H. Osborn and A. Petkou, Implications of conformal invariance in field theories for general dimensions, Annals Phys. 231 (1994) 311-362, hep-th/9307010.

[17] K. Behrndt, M. Cvetic, and W. Sabra, Nonextreme black holes of five-dimensional N=2 AdS supergravity, Nucl.Phys. B553 (1999) 317-332, hep-th/9810227.

[18] D. T. Son and A. O. Starinets, Hydrodynamics of R-charged black holes, JHEP 0603 (2006) 052, hep-th/0601157.

[19] A. Batrachenko, J. T. Liu, R. McNees, W. Sabra, and W. Wen, Black hole mass and Hamilton-Jacobi counterterms, JHEP 0505 (2005) 034, hep-th/0408205. 
[20] D. Marolf and S. F. Ross, Boundary Conditions and New Dualities: Vector Fields in AdS/CFT, JHEP 0611 (2006) 085, hep-th/0606113.

[21] E. Witten, Multitrace operators, boundary conditions, and AdS / CFT correspondence, hep-th/0112258.

[22] M. Berkooz, A. Sever, and A. Shomer, 'Double trace' deformations, boundary conditions and space-time singularities, JHEP 0205 (2002) 034, hep-th/0112264.

[23] M. Duff, TASI lectures on branes, black holes and Anti-de Sitter space, hep-th/9912164. Based on talks delivered at the Theoretical Advanced Study Institute, Boulder, Colorado, Jun 1999 and the Banff Summer School, Alberta, Canada, Jun-Jul 1999.

[24] N. Bobev, N. Halmagyi, K. Pilch, and N. P. Warner, Holographic, N=1 Supersymmetric RG Flows on M2 Branes, JHEP 0909 (2009) 043, arXiv:0901.2736.

[25] A. Donos and J. P. Gauntlett, Superfluid black branes in $A d S_{4} \times S^{7}$, JHEP 1106 (2011) 053, arXiv:1104.4478. * Temporary entry*.

[26] D. T. Son and P. Surowka, Hydrodynamics with Triangle Anomalies, Phys.Rev.Lett. 103 (2009) 191601, arXiv:0906.5044. 